\title{
A IMPORTÂNCIA DE UNIDADES DE CONSERVAÇÃO NA PROTEÇÃO DE AVES DA FLORESTA ATLÂNTICA FRENTE Ả FRAGMENTAÇÃO
}

\author{
ZIMMERMANN, C.E. ${ }^{*}$; BRANCO, J.O.; MARENZI, R.C..; WILLRICH, M.A.K. ${ }^{2}$ \\ 1. Departamento de Engenharia Florestal (FURB) \\ 2. Escola do Mar, Ciência e Tecnologia (UNIVALI) \\ *Corresponding author: cezimmer@furb.br
}

\begin{abstract}
Zimmermann, C.E., Branco, J.O., Marenzi, R.C., Willrich, M.A.K. (2020). A importância de unidades de conservação na proteção de aves da floresta atlântica frente à fragmentação. Braz. J. Aquat. Sci. Technol. 24(1). elSSN 1983-9057. DOI: 15198/bjast.v24n1. Due to the few studies in ornithology in the gleba menor of the Sassafras and Canela Preta Biological, the objective of this study was to evaluate the role of these Units for the preservation of forest birds. In the field, a monthly quantitative inventory was carried out, covering all seasons and periods of the day. The specimens were identified by sonic and visual evidences using a $7 \times 35 \mathrm{~mm}$ binocular. The displacement in each area was one hour, carried out by walking at a constant speed $( \pm 3.0 \mathrm{~km} / \mathrm{h})$ on pre-existing tracks, covering the largest area and possible environments. For each unit with the area and perimeter values, the form factor was calculated. With a sample effort of 65 and 71 hours of observation for the Canela Preta Biological Reserve and for the gleba menor, it was possible to record 182 and 190 species respectively. The richness in the areas can be considered high when compared to the richness in the greater area in the Sassafras Rebio (3,840 ha), where 237 species were registered. Among the species are those considered endemic, which for the two areas add up to 84 species, such as Lipaugus lanioides (Lesson, 1844). It was possible to record the presence of species characteristic of altitudes such as: Pyrrhocoma ruficeps (Strickland, 1844). Among species of conservation interest we have Amazona vinacea (Kuhl, 1820) considered threatened (EN). The most representative species was the Critically Endangered (CR) Primolius maracana (Vieillot, 1816). For the shape factor we obtained values of 0.21 (Sassafras) and 0.18 (Canela-Preta). Values less than 1 indicate a more circular area and, consequently, less edge effect. From the above, it is evident the importance of these protected areas for the conservation of bird species against the fragmentation of the Atlantic Forest in Santa Catarina.
\end{abstract}

Key words: Conservation Units, Atlantic Forest, Fragmentation, Birds.

\section{INTRODUÇÃO}

\section{O conhecimento ornitológico catarinense}

Um dos primeiros trabalhos da avifauna catarinense foi apresentado com a publicação da Lista preliminar das aves do Estado de Santa Catarina (Sicketal. 1979a). Na sequência, por iniciativa do governo do estado, élançadaa Lista preliminar das aves existentes nos Parques e Reservas Biológicas de Santa Catarina, com 521 espécies de aves apresentadas (Sick et al.,1979b).

O trabalho mais sistemático sobre as aves catarinenses aparece no livro Aves do Estado de Santa Catarina (Sick et al., 1981), onde é apresentado um breve resumo dos aspectos históricos da ornitologia catarinense desde o século XVI. Também se destacam os estudos do conde Hans von Berlepsch que publicou trabalhos sobre aves de uma coleção com 144 espécies que foram coletadas onde hoje é o Parque Botânico Morro do Baú em Ilhota, na época colônia Blumenau.

Novas informações sobre as aves de Santa Catarina são apresentadas por Sick \& Bege (1984), com a apresentação de 10 novas espécies, além de dados complementares para outras espécies. Sick et al. (1988) apresentam dados sobres as aves do município de Antônio Carlos. Bege \& Pauli (1989) ampliaram a lista das espécies catarinense com o registro de Morus serrator (Gray, 1843). Para o Sul do Estado são apresentados aspectos da conservação de 238 espécies (Bege \& Marterer, 1991). Com relação às aves oceânicas ou costeiras, destacamos os vários trabalhos ao longo da costa catarinense, abrangendo vários aspectos da ecologia das aves marinhas (Soares \& Schiffer, 1995 a,b; Branco, 2000; 2001; 2003; 2004; 2005).

O mais completo trabalho sobre as aves catarinense com a apresentação de mapas de distribuição e ocorrência de 596 espécies é apresentado por Rosário (1996). Em levantamentos mais recentes a riqueza de aves está próxima de 650 spp. (Pacheco \& Laps, 2001; Rupp et al., 2007; 2008).

Para o Vale do Itajaí os estudos se concentram nas espécies em Unidades de Conservação e fragmentos florestais (Zimmermann, 1989, 1992, 1993, 1995, 1999; Marterer, 1996; Roos, 2002; Krieck, 2006; Zimmermann \& Branco; 2009 e Guztzazky et al., 2014).

Em Santa Catarina os impactos da fragmentação florestal sobre a comunidade de aves são pouco estudados. Existem lacunas no conhecimento ornitológico que pode comprometer, por exemplo, avaliações da ecologia populacional e de conservação 
das espécies (Straube et al., 2004).

O domínio da Floresta Atlântica, onde na Bacia do Itajaí encontramos a tipologia Floresta Ombrófila Densa, é reconhecido internacionalmente como um Hotspot, ou seja, uma área com elevada taxa de endemismo, sofrendo ameaças com altas taxas de perda de habitats. Apesar disso, os níveis de exploração florestal em parte pela expansão do agronegócio e das cidades, acarretaram uma expressiva redução de habitats na Floresta Atlântica cuja área remanescente pode ser inferior a $10 \%$ da área original (Myers et al., 2000; Ribeiro et al., 2009).

A fragmentação de remanescentes florestais é considerada uma importante força que acarreta perdas de biodiversidade (Bierregaard \& Lovejoy, 1989), refletindo na composição das comunidades e podendo levar a extinção de populações residentes e que dependem de ambientes conservados para a manutenção de populações viáveis (Goerck, 1997; Pizo, 1997).

Derivada da Teoria de Biogeografia de Ilhas, ocorre forte relação espécie-área, onde fragmentos maiores e menos isolados abrigariam um maior número de espécies (MacArthur \& Wilson, 1963, 1967; Debinski \& Holt 2000, Laps et al., 2003), e cujos impactos dependem do histórico de perturbação, desenho e grau de isolamento, além da complexidade da matriz entre fragmentos, tornando as populações menos suscetíveis a eventos demográficos e ambientais aleatórios (Bierregaard et al., 1992; Tabanez et al., 1997; Pardini, 2004, Boscolo \& Metzger, 2009; Boscolo \& Metzger, 2011)

Dentro desse contexto, o objetivo desde estudo foi caracterizar a comunidade de aves das Reservas Biológicas (ReBio) Estadual da Canela Preta e a Reserva Biológica Estadual do Sassafrás (Gleba Menor), buscando subsidiar ações práticas de gestão de unidades de conservação (UC) e embasar estratégias de conservação com enfoque na Ecologia de Paisagem.

\section{MATERIAL E MÉTODOS}

\section{Áreas de estudo}

A Reserva Biológica Estadual do Sassafrás está inserida na Bacia Hidrográfica do Itajaí, fazendo parte de um conjunto de áreas protegidas (Figura 01). Criada pelo Decreto no 2.221/77, possui uma área de 5.229 hectares, dividida em duas glebas. A gleba menor, objeto desse trabalho, possui 1.361 hectares e está localizada no município de Benedito Novo. No seu Plano de Manejo as informações sobre a comunidade de aves dessa gleba são ainda incompletas e insuficientes para o manejo desta parte da UC (Fundação do Meio Ambiente, 2010). A Reserva Biológica Estadual da Canela Preta estende-se pelos municípios de Botuverá e Nova Trento. Criada junho de 1980 pelo decreto

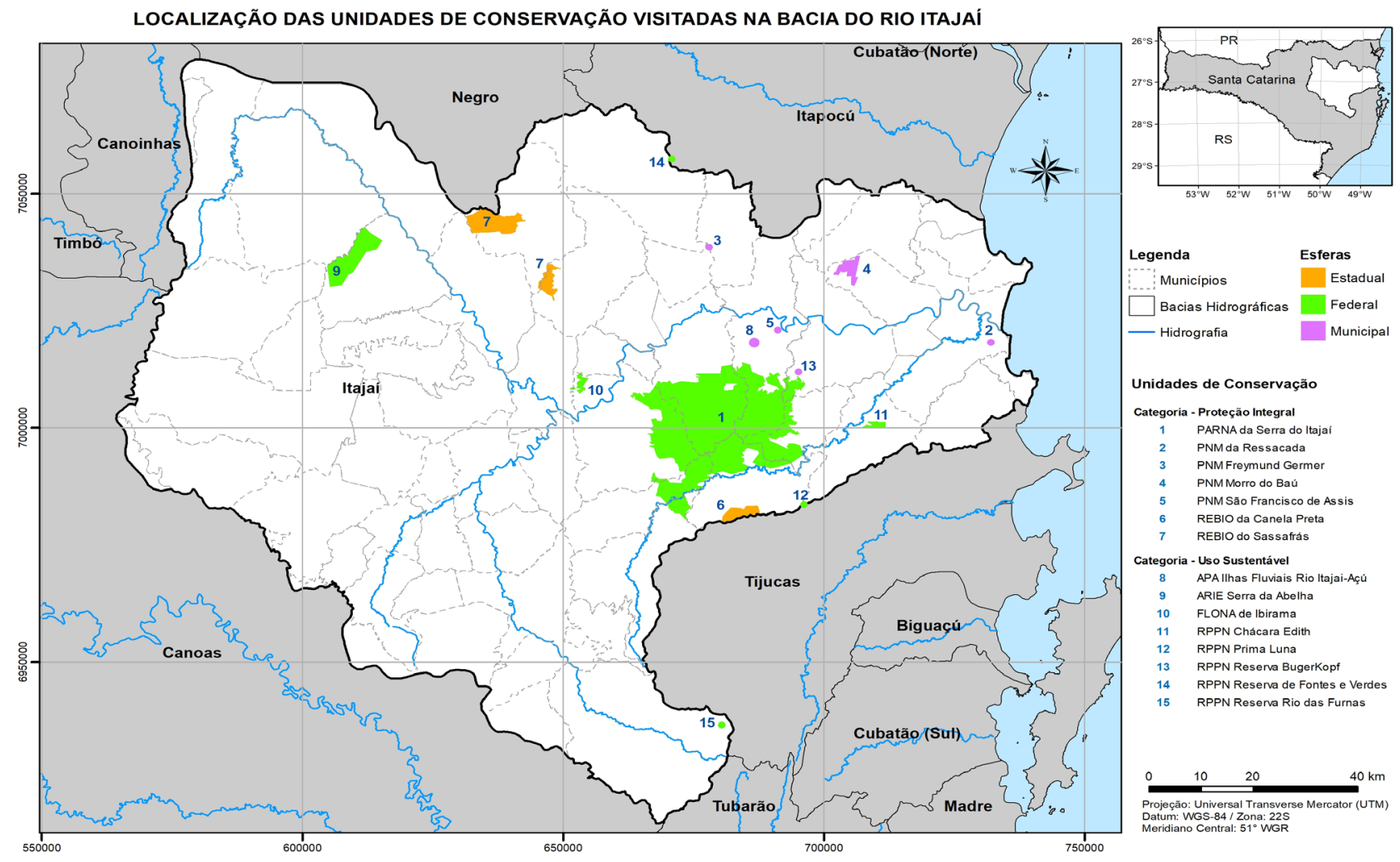

Figura 1 - Localização das unidades de conservação na Bacia Hidrográfica do Itajaí, sendo áreas de estudo: Rebio da Canela-preta (6), Rebio do Sassafrás, gleba maior e menor (7). Fonte. Inventario Florístico Florestal de Santa Catarina- IFFSC/FURB). 
11.232 possui área de 1.899 hectares e ainda não possui plano de manejo (Figura 01).

\section{Análise dos dados}

As comunidades de aves em cada Reserva Biológica foram caracterizadas pelo cálculo de descritores ecológicos (Matarazzo-Neuberger, 1995; D’AngeloNeto et al., 1998; Zimmermann \& Branco, 2009; Brummelhaus et al., 2012; Guztzazky et al., 2014), considerando: a) Índice de Diversidade de Shannon $\left(H^{\prime}\right)$, que permite comparar o grau de heterogeneidade das áreas estudadas, com base na abundância proporcional das espécies da comunidade, $\mathrm{H}^{\prime}=-\sum$ (pilnpi) onde pi = proporção dos indivíduos da espécie i em relação ao número total de indivíduos da comunidade; b) Índice de Equidade (J), que avalia o grau de dominância das espécies na comunidade, $\mathrm{J}=\mathrm{H}^{\prime} /$ Hmax, onde $\mathrm{H}^{\prime}$ é o índice de Shannon e Hmax é o máximo valor de $\mathrm{H}^{\prime}$, sendo $\mathrm{Hmax}=\log$ de $\mathrm{Si}$, onde Si o número de espécies do fragmento.

O papel das Reservas Biológica Estaduais na conservação das aves foi avaliado pela comparação da riqueza específica encontrada, com a riqueza de espécies de aves da gleba maior da Rebio do Sassafrás e do Parque Nacional da Serra do Itajaí, maior Unidade de Conservação da Bacia do Itajaí. Para avaliar se os métodos empregados foram eficientes para registrar o maior número de espécies de aves das Reservas Biológicas foi construído o gráfico do número acumulado de espécies, que avalia o esforço amostral (Straube \& Bianconi, 2002).

Com os dados de área e perímetro das unidades de conservação, obtidos dos respectivos planos de manejo, foram calculados o Fator de Forma por meio da fórmula adaptada de Marenzi \& Roderjan (2005), conforme segue:

$$
\text { Fator de forma }=P / 2 \pi(\sqrt{ } \mathrm{A} / \sqrt{ } \pi) \quad(\text { Equação } 1)
$$

Onde $\mathrm{P}=$ perímetro; $\mathrm{A}$ = área do fragmento.

\section{RESULTADOS E DISCUSSÕES}

Os trabalhos de campo resultaram em um esforço amostral de 60 horas de observação para Reserva Biológica da Canela Preta e de 75 horas para a Gleba Menor da Reserva Biológica do Sassafrás, possibilitando no registro de 182 e 190 espécies de aves, respectivamente (Figura 02; Tabela 01).

A primeira inciativa para se conhecer a comunidade de aves da gleba menor da Reserva Biológica Estadual do Sassafrás ocorreu através de estudos realizados no final da década de $70 \mathrm{com}$ a publicação da lista preliminar das aves encontradas em parques e reservas biológicas em Santa Catarina

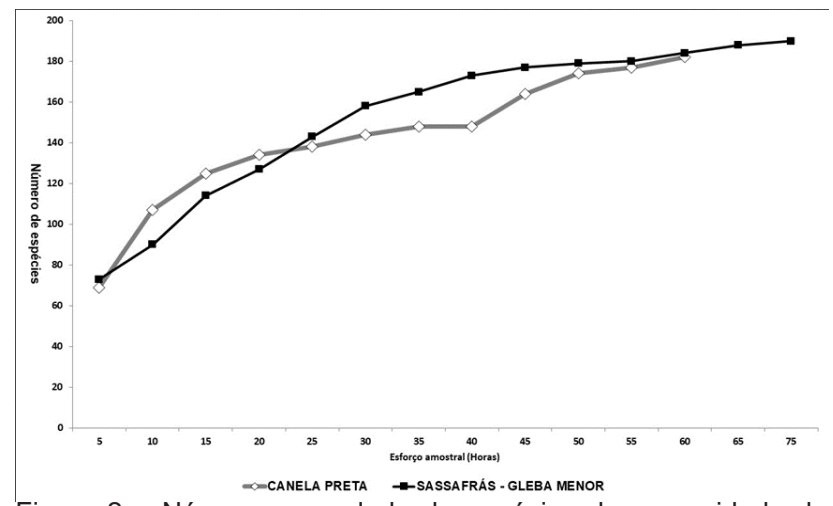

Figura 2 - Número acumulado de espécies da comunidade de aves da Reserva Biológica Estadual da Canela Preta e da Reserva Biológica Estadual do Sassafrás, Gleba Menor. Santa Catarina.

(Sick etal., 1979b), onde os autores listaram 38 espécies, além de novos registros de espécies relevantes apresentados por Rosário (1996).

A riqueza de espécies de aves encontrada na gleba menor da Rebio do Sassafrás (190) é importante, pois representa $80,17 \%$ das aves registradas para o interior da gleba maior da Reserva Biológica, onde foram registradas 237 espécies durante os estudos para a elaboração do Plano de Manejo da UC (Fundação do Meio Ambiente, 2010).

Entre as espécies de aves registradas na gleba menor, um total de 36 não foram registradas na gleba maior, sendo elas: Crypturellus tataupa (Temminck, 1815), Amazonetta brasiliensis (Gmelin, 1789), Nannopterumbrasilianus (Gmelin, 1789), Butorides striata (Linnaeus, 1758), Syrigma sibilatrix (Temminck, 1824), Sarcoramphus papa (Linnaeus, 1758), Gallinula galeata (Lichtenstein, 1818), Falco sparverius (Linnaeus, 1758), Patagioenas cayennensis (Bonnaterre, 1792), Zenaida auriculata (Des Murs, 1847), Guira guira (Gmelin, 1788), Cypseloides senex (Temminck, 1826), Lophornis chalybeus (Vieillot, 1822), Amazilia versicolor (Vieillot, 1818), Megaceryle torquata (Linnaeus, 1766), Chloroceryle amazona (Latham, 1790), Chloroceryle americana (Gmelin, 1788), Melanerpes candidus (Otto, 1796), Hypoedaleus guttatus (Vieillot, 1816), Cichlocolaptes leucophrus (Jardine \& Selby, 1830), Manacus manacus (Linnaeus, 1766), Myiornis auricularis (Vieillot, 1818), Elaenia parvirostris (Pelzeln, 1868), Attila rufus (Vieillot, 1819), Colonia colonus (Vieillot, 1818), Satrapa icterophrys (Vieillot, 1818), Turdus leucomelas (Vieillot, 1818), Mimus saturninus (Lichtenstein, 1823), Tangara palmarum (Wied, 1821), Tangara ornata (Sparrman, 1789), Conirostrum speciosum (Temminck, 1824), Tiaris fuliginosus (Wied, 1830), Molothrus rufoaxillaris (Cassin, 1866), Gnorimopsar chopi (Vieillot, 1819), Pseudoleistes guirahuro (Vieillot, 1819) e Euphonia cyanocephala (Vieillot, 1818).

Considerando o registro de Phylloscartes 
difficilis (Ihering \& Ihering, 1907) para a gleba menor, apresentado no plano de manejo da Rebio do Sassafrás

(Funação do Meio Ambiente, 2010), temos uma riqueza total de 274 espécies de aves para esta Reserva Biológica.

A riqueza de aves da Rebio da Canela Preta (182) é da mesma forma representativa, quando comparada com a riqueza em unidades de conservação com áreas bem superiores, dentro do domínio da Floresta Atlântica, como o Parque Nacional da Serra do Itajaí (57 mil ha) com 320 espécies registradas (Zimmermann et al., 2018), o Parque Estadual da Serra do Tabuleiro (83 mil ha), onde foram observados 270 espécies de aves (Albuquerque \& Brüggemann, 1996), bem como o Parque Nacional do Iguaçu (185 mil ha) com 317 espécies de aves (Straube \& Urben-Filho, 2004).

Para a Rebio da Canela Preta registramos treze espécies de aves exclusivas, sendo elas: Penelope superciliaris Temminck, 1815, Gallinago paraguaiae (Boddaert, 1783) Ramphodon naevius (Dumont, 1818), Florisuga fusca (Vieillot, 1817), Myrmotherula unicolor (Ménétriès, 1835), Dysithamnus stictothorax (Temminck, 1823), Herpsilochmus rufimarginatus (Temminck, 1822), Terenura maculata (Wied, 1831), Drymophila ochropyga (Hellmayr, 1906), Hemitriccus orbitatus (Wied, 1831), Phyllomyias griseocapilla Sclater, 1862, Orthogonys chloricterus (Vieillot, 1819), Chlorophanes spiza (Linnaeus, 1758).

Desta forma, a riqueza total nas UCs estudadas sobe para 287 espécies, o que representa $48,157 \%$ das espécies de aves para Santa Catarina, conforme lista apresentada por Rosário (1996).

Com relação ao número médio de espécies e indivíduos, registramos uma média de 32,76 $(D p=5,09)$ espécies e 73,27 (Dp = 11,91) indivíduos por hora de observação, respectivamente, para a Gleba Menor da Rebio do Sassafrás. Para a Reserva Biológica da Canela-Preta o número médio de espécies e indivíduos ficou em 37,9 (Dp =6,99) espécies e 89,11 $(\mathrm{Dp}=16,73)$ indivíduos, respectivamente.

Para os valores médios de diversidade e equidade da comunidade de aves, registramos 3,29 nats/ind. e 0,95 para a gleba menor da Rebio do Sassafrás e 3,41 nats/ind. e 0,95 para a Rebio da Canela-preta, sugerindo uma comunidade de aves rica e sem a presença de espécies dominantes, representada por espécies com uma elevada densidade.

Valores de diversidade geralmente variam em torno de 1,8 e 5,2 nats/ind., onde nas regiões tropicais este índice tende a ser superior a 2,5 nats/ind. (Machado, 1996). Valores semelhantes foram registrados em estudos de fragmentos florestais no estado do Espirito Santo, com a diversidade oscilando entre 3,58 e 3,93 nats/ind., dependendo do tamanho do fragmento
(Marsden et al., 2001). Valores similares foram obtidos em estudos em fragmentos florestais na região do Vale do Itajaí (Guztzazky et al., 2014). Em oito pequenos fragmentos na área urbana da cidade de Blumenau, Cruz e Zimmermann (2011) obtiveram valores de diversidade entre 2,94 e 3,39 nats/ind. No Parque Natural São Francisco de Assis, localizado na área central do Município de Blumenau, foram registrados valores de diversidade oscilando em torno de 3,38 nats/ind. com uma equidade de 0,93 (Zimmermann, 1999).

Com relação ao fator de forma obtivemos valores de 0,20 para a gleba menor da Rebio do Sassafrás e 0,13 para a Rebio da Canela Preta. Valores menores para a Rebio da Canela Preta indicam que está possui uma área mais circular e, consequentemente, com um menor efeito de borda, condição considerada importante para a conservação das espécies (Zimmermann et al., 2017), uma vez que pode gerar maior integridade ecológica, quando a matriz (normalmente entorno dos fragmentos) é urbanizada ou alterada por atividades impactantes.

Quando somamos a zona de amortecimento da gleba menor da Rebio do Sassafrás, os valores de área e perímetro sobem para 3871,54 hectares e $4 \mathrm{~km}$, respectivamente, resultando na redução do fator de forma para 0,18. Essa relação pode estar indicando a importância de se buscar a ampliação das UCs em termos de área em relação ao seu perímetro, aumentando o seu valor de conservação (Marenzi \& Roderjan, 2005).

Entre as espécies de interesse conservacionista, temos aquelas consideradas endêmicas da Floresta Atlântica, como Lipaugus lanioides (Lesson, 1844), que totalizam 102 espécies considerando a Rebio Canela Preta e as duas glebas da Rebio do Sassafrás. As UCs apresentam altitudes acima de 600 metros acima do nível do mar, o que torna possível ambientes para o registro de espécies de aves características destas altitudes como: Carpornis cucullata (Swainson, 1821), Heliodoxa rubricauda (Boddaert, 1783) e Pyrrhocoma ruficeps (Strickland, 1844).

Considerando as três áreas, as duas glebas da Reserva Biológica do Sassafrás e a Reserva Biológica da Canela Preta, registramos 15 espécies com algum grau de ameaçada para Santa Catarina, segundo CONSEMA (2011). Na categoria vulnerável temos 10 espécies, onde destacamos Triclaria malachitacea (Spix, 1824). Entre as quatro espécies consideradas ameaçadas, temos o registro de Amazona vinacea (Kuhl, 1820), com nove indivíduos observados. Contudo, a espécie mais representativa foi Primolius maracana (Vieillot, 1816), considerada criticamente ameaçada. Fato que comprova a importância das UCs estudadas e a necessidade de efetividade na gestão. 
Tabela 1 - Avifauna das Reservas Biológicas do Sassafrás, Glebas Maior e Menor, e Rebio da Canela Preta. Famílias e espécies de acordo com Piacentini et al. (2015); Aves endêmicas (End.) e Status de Conservação (S.C.) de acordo com CONSEMA (2011) E IUCN (2017), onde: LC: Pouco Preocupante; NT: quase-ameaçado; VU: vulnerável; EN: Ameaçado, CR: Criticamente ameaçado. END: espécie considerada endêmica do Bioma Floresta Atlântica.

\begin{tabular}{|c|c|c|c|c|c|c|}
\hline Nome do Taxon & $\begin{array}{c}\text { Rebio } \\
\text { Sassafrás }\end{array}$ & $\begin{array}{l}\text { Gleba } \\
\text { Menor }\end{array}$ & $\begin{array}{c}\text { Rebio } \\
\text { Canela } \\
\text { Preta }\end{array}$ & END & IUCN -SC & Nome comum \\
\hline Tinamus solitarius (Vieillot, 1819) & 1 & 0 & 1 & 1 & NT - VU & Macuco \\
\hline $\begin{array}{c}\text { Crypturellus obsoletus } \\
\text { (Temminck, 1815) }\end{array}$ & 1 & 1 & 1 & - & LC - LC & inhambuguaçu \\
\hline $\begin{array}{l}\text { Crypturellus noctivagus (Wied, } \\
\text { 1820) }\end{array}$ & 1 & 0 & 0 & & NT - EN & jaó-do-sul \\
\hline $\begin{array}{l}\text { Crypturellus tataupa (Temminck, } \\
\text { 1815) }\end{array}$ & 0 & 1 & 1 & - & LC - LC & inhambu-chintã \\
\hline $\begin{array}{c}\text { Rhynchotus rufescens } \\
\text { (Temminck, 1815) }\end{array}$ & 1 & 0 & 0 & - & LC - LC & Perdiz \\
\hline $\begin{array}{c}\text { Cairina moschata (Linnaeus, } \\
\text { 1758) }\end{array}$ & 1 & 0 & 0 & - & LC - LC & pato-do-mato \\
\hline $\begin{array}{l}\text { Amazonetta brasiliensis (Gmelin, } \\
1789)\end{array}$ & 0 & 1 & 1 & - & LC - LC & pé-vermelho \\
\hline Ortalis squamata (Lesson, 1829) & 1 & 0 & 0 & 1 & LC - LC & aracuã \\
\hline $\begin{array}{c}\text { Penelope superciliaris } \\
\text { (Temminck, 1815) }\end{array}$ & 0 & 0 & 1 & - & LC - VU & jacupemba \\
\hline $\begin{array}{c}\text { Penelope obscura (Temminck, } \\
\text { 1815) }\end{array}$ & 1 & 1 & 1 & - & LC - LC & jacuaçu \\
\hline $\begin{array}{l}\text { Odontophorus capueira (Spix, } \\
\text { 1825) }\end{array}$ & 1 & 1 & 1 & 1 & LC - LC & uru \\
\hline $\begin{array}{c}\text { Nannopterum brasilianus } \\
\text { (Gmelin, 1789) }\end{array}$ & 0 & 1 & 0 & - & LC - LC & biguá \\
\hline $\begin{array}{c}\text { Butorides striata (Linnaeus, } \\
1758 \text { ) }\end{array}$ & 0 & 1 & 0 & - & LC - LC & socozinho \\
\hline $\begin{array}{c}\text { Syrigma sibilatrix (Temminck, } \\
1824)\end{array}$ & 0 & 1 & 1 & - & LC - LC & maria-faceira \\
\hline $\begin{array}{l}\text { Theristicus caudatus (Boddaert, } \\
\text { 1783) }\end{array}$ & 1 & 1 & 1 & - & LC - LC & curicaca \\
\hline Cathartes aura (Linnaeus, 1758) & 1 & 1 & 1 & - & LC - LC & $\begin{array}{l}\text { urubu-de-cabeça- } \\
\text { vermelha }\end{array}$ \\
\hline $\begin{array}{c}\text { Coragyps atratus (Bechstein, } \\
1793)\end{array}$ & 1 & 1 & 1 & - & LC - LC & urubu-de-cabeça-preta \\
\hline $\begin{array}{c}\text { Sarcoramphus papa (Linnaeus, } \\
1758 \text { ) }\end{array}$ & 0 & 1 & 1 & - & LC - LC & Urubu-rei \\
\hline $\begin{array}{l}\text { Elanoides forficatus (Linnaeus, } \\
\qquad 1758 \text { ) }\end{array}$ & 1 & 1 & 1 & - & LC - LC & gavião-tesoura \\
\hline Elanus leucurus (Vieillot, 1818) & 1 & 0 & 0 & - & LC - LC & gavião-peneira \\
\hline $\begin{array}{c}\text { Pandion haliaetus (Linnaeus, } \\
\text { 1758) }\end{array}$ & 1 & 0 & 0 & - & LC -LC & Águia-pescadora \\
\hline $\begin{array}{l}\text { Harpagus diodon (Temminck, } \\
\text { 1823) }\end{array}$ & 1 & 0 & 1 & - & LC - LC & gavião-bombachinha \\
\hline Ictinia plumbea (Gmelin, 1788) & 1 & 0 & 0 & - & LC - LC & gaviao \\
\hline $\begin{array}{c}\text { Accipiter poliogaster (Temminck, } \\
1824)\end{array}$ & 1 & 0 & 0 & - & NT - LC & tauató-pintado \\
\hline Accipiter striatus (Vieillot, 1808) & 1 & 0 & 0 & - & LC - LC & gavião-miúdo \\
\hline
\end{tabular}




\begin{tabular}{|c|c|c|c|c|c|c|}
\hline Nome do Taxon & $\begin{array}{c}\text { Rebio } \\
\text { Sassafrás }\end{array}$ & $\begin{array}{l}\text { Gleba } \\
\text { Menor }\end{array}$ & $\begin{array}{c}\text { Rebio } \\
\text { Canela } \\
\text { Preta }\end{array}$ & END & IUCN -SC & Nome comum \\
\hline Accipiter bicolor (Vieillot, 1817) & 1 & 0 & 0 & - & LC - LC & $\begin{array}{l}\text { gavião-bombachinha- } \\
\text { grande }\end{array}$ \\
\hline $\begin{array}{l}\text { Urubitinga urubitinga (Gmelin, } \\
\text { 1788) }\end{array}$ & 1 & 0 & 0 & - & LC - LC & Gavião-preto \\
\hline $\begin{array}{l}\text { Pseudastur polionotus (Kaup, } \\
\text { 1847) }\end{array}$ & 1 & 0 & 1 & 1 & NT - LC & gavião-pombo-grande \\
\hline $\begin{array}{c}\text { Parabuteo leucorrhous (Quoy \& } \\
\text { Gaimard, 1824) }\end{array}$ & 1 & 1 & 0 & - & LC - LC & gavião-de-sobre-branco \\
\hline $\begin{array}{l}\text { Rupornis magnirostris (Gmelin, } \\
1788 \text { ) }\end{array}$ & 1 & 1 & 1 & - & LC - LC & Gaviao-Carijó \\
\hline Buteo brachyurus Vieillot, 1816 & 1 & 0 & 1 & - & LC - LC & gavião-de-cauda-curta \\
\hline Spizaetus tyrannus (Wied, 1820) & 1 & 0 & 0 & - & LC - VU & gavião-pega-macaco \\
\hline $\begin{array}{c}\text { Spizaetus melanoleucus (Vieillot, } \\
1816)\end{array}$ & 1 & 0 & 1 & - & LC - EN & gavião-pato \\
\hline Aramides saracura (Spix, 1825) & 1 & 1 & 1 & 1 & LC - LC & saracura-do-mato \\
\hline $\begin{array}{c}\text { Gallinula galeata (Lichtenstein, } \\
1818)\end{array}$ & 0 & 1 & 0 & - & LC - LC & frango-d'água-comum \\
\hline Caracara plancus (Miller, 1777) & 1 & 1 & 1 & - & LC - LC & caracará \\
\hline $\begin{array}{l}\text { Milvago chimachima (Vieillot, } \\
\text { 1816) }\end{array}$ & 1 & 1 & 0 & - & LC - LC & carrapateiro \\
\hline Micrastur ruficollis (Vieillot, 1817) & 1 & 1 & 1 & - & LC - LC & falcão-caburé \\
\hline $\begin{array}{c}\text { Micrastur semitorquatus (Vieillot, } \\
1817 \text { ) }\end{array}$ & 1 & 0 & 0 & - & LC - LC & falcão-relógio \\
\hline $\begin{array}{c}\text { Falco sparverius (Linnaeus, } \\
\text { 1758) }\end{array}$ & 0 & 1 & 1 & - & LC - LC & quiriquiri \\
\hline Vanellus chilensis (Molina, 1782) & 1 & 1 & 1 & - & LC - LC & quero-quero \\
\hline $\begin{array}{l}\text { Gallinago paraguaiae (Boddaert, } \\
\text { 1783) }\end{array}$ & 0 & 0 & 1 & - & LC - LC & narcejão \\
\hline $\begin{array}{c}\text { Columbina talpacoti (Temminck, } \\
\text { 1811) }\end{array}$ & 1 & 1 & 1 & - & LC - LC & rolinha-roxa \\
\hline $\begin{array}{c}\text { Claravis pretiosa (Ferrari-Perez, } \\
1886)\end{array}$ & 1 & 0 & 0 & - & LC - LC & pararu-azul \\
\hline $\begin{array}{c}\text { Patagioenas speciosa (Gmelin, } \\
\text { 1789) }\end{array}$ & 1 & 0 & 0 & & LC - LC & Pomba-trocal \\
\hline $\begin{array}{l}\text { Patagioenas picazuro } \\
\text { (Temminck, 1813) }\end{array}$ & 1 & 1 & 0 & - & LC - LC & pombão \\
\hline $\begin{array}{l}\text { Patagioenas cayennensis } \\
\quad \text { (Bonnaterre, 1792) }\end{array}$ & 0 & 1 & 0 & - & LC - LC & pomba-galega \\
\hline $\begin{array}{c}\text { Patagioenas plumbea (Vieillot, } \\
\text { 1818) }\end{array}$ & 1 & 1 & 1 & - & LC - LC & pomba-amargosa \\
\hline $\begin{array}{c}\text { Zenaida auriculata (Des Murs, } \\
1847 \text { ) }\end{array}$ & 0 & 1 & 0 & - & LC - LC & pomba-de-bando \\
\hline $\begin{array}{l}\text { Leptotila verreauxi (Bonaparte, } \\
\qquad 1855)\end{array}$ & 1 & 1 & 1 & - & LC - LC & juriti-pupu \\
\hline $\begin{array}{c}\text { Leptotila rufaxilla (Richard \& } \\
\text { Bernard, 1792) }\end{array}$ & 1 & 0 & 0 & - & LC - LC & juriti-gemedeira \\
\hline $\begin{array}{c}\text { Geotrygon montana (Linnaeus, } \\
1758 \text { ) }\end{array}$ & 1 & 0 & 1 & - & LC - LC & pariri \\
\hline
\end{tabular}




\begin{tabular}{|c|c|c|c|c|c|c|}
\hline Nome do Taxon & $\begin{array}{c}\text { Rebio } \\
\text { Sassafrás }\end{array}$ & $\begin{array}{l}\text { Gleba } \\
\text { Menor }\end{array}$ & $\begin{array}{c}\text { Rebio } \\
\text { Canela } \\
\text { Preta } \\
\end{array}$ & END & IUCN -SC & Nome comum \\
\hline $\begin{array}{l}\text { Primolius maracana (Vieillot, } \\
\text { 1816) }\end{array}$ & 1 & 1 & 0 & - & NT - CR & maracanã-verdadeira \\
\hline $\begin{array}{c}\text { Psittacara leucophthalmus } \\
\text { (Statius Muller, 1776) }\end{array}$ & 1 & 0 & 0 & - & LC - LC & periquitão-maracanã \\
\hline Pyrrhura frontalis (Vieillot, 1817) & 1 & 1 & 1 & 1 & LC - LC & tiriba-de-testa-verm elha \\
\hline $\begin{array}{l}\text { Forpus xanthopterygius (Spix, } \\
\text { 1824) }\end{array}$ & 1 & 1 & 1 & - & LC - LC & Tuim \\
\hline Brotogeris tirica (Gmelin, 1788) & 1 & 1 & 1 & 1 & LC - LC & periquito-rico \\
\hline $\begin{array}{c}\text { Pionopsitta pileata (Scopoli, } \\
\text { 1769) }\end{array}$ & 1 & 1 & 1 & 1 & LC - LC & cuiú-cuiú \\
\hline Pionus maximiliani (Kuhl, 1820) & 1 & 0 & 1 & - & LC - LC & maitaca-verde \\
\hline Amazona vinacea (Kuhl, 1820) & 1 & 1 & 0 & 1 & VU - EN & papagaio-de-peito-roxo \\
\hline $\begin{array}{c}\text { Triclaria malachitacea (Spix, } \\
\text { 1824) }\end{array}$ & 1 & 1 & 1 & 1 & NT - VU & sabiá-cica \\
\hline Piaya cayana (Linnaeus, 1766) & 1 & 1 & 1 & - & LC - LC & alma-de-gato \\
\hline Crotophaga ani (Linnaeus, 1758) & 0 & 0 & 1 & - & LC - LC & anu-preto \\
\hline Guira guira (Gmelin, 1788) & 0 & 1 & 1 & - & LC - LC & anu-branco \\
\hline Tapera naevia (Linnaeus, 1766) & 1 & 1 & 0 & - & LC - LC & Saci \\
\hline Tyto furcata (Temminck, 1827) & 1 & 0 & 0 & - & LC - LC & coruja-da-igreja \\
\hline $\begin{array}{c}\text { Megascops choliba (Vieillot, } \\
\text { 1817) }\end{array}$ & 1 & 0 & 0 & - & LC - LC & corujinha-do-mato \\
\hline $\begin{array}{c}\text { Pulsatrix koeniswaldiana (Bertoni } \\
\text { \& Bertoni, 1901) }\end{array}$ & 1 & 0 & 1 & 1 & LC - LC & murucututu \\
\hline Strix hylophila (Temminck, 1825) & 1 & 0 & 0 & 1 & NT - LC & coruja-listrada \\
\hline Nyctibius griseus (Gmelin, 1789) & 1 & 0 & 0 & - & LC - LC & mãe-da-lua \\
\hline $\begin{array}{l}\text { Hydropsalis torquata (Gmelin, } \\
\text { 1789) }\end{array}$ & 1 & 0 & 0 & - & LC - LC & bacurau-tesoura \\
\hline $\begin{array}{l}\text { Hydropsalis forcipata (Nitzsch, } \\
\text { 1840) }\end{array}$ & 1 & 0 & 0 & - & LC - LC & bacurau-tesoura-gigante \\
\hline $\begin{array}{l}\text { Lurocalis semitorquatus (Gmelin, } \\
\text { 1789) }\end{array}$ & 1 & 0 & 0 & - & LC - LC & Tuju \\
\hline $\begin{array}{c}\text { Antrostomus sericocaudatus } \\
\text { (Cassin, 1849) }\end{array}$ & 1 & 0 & 0 & - & LC - LC & bacurau-rabo-de-seda \\
\hline $\begin{array}{l}\text { Cypseloides fumigatus (Streubel, } \\
\text { 1848) }\end{array}$ & 1 & 0 & 0 & - & $\mathrm{LC}-\mathrm{LC}$ & taperuçu-preto \\
\hline $\begin{array}{c}\text { Cypseloides senex (Temminck, } \\
\text { 1826) }\end{array}$ & 0 & 1 & 0 & - & LC - LC & taperuçu-velho \\
\hline $\begin{array}{c}\text { Streptoprocne zonaris (Shaw, } \\
1796)\end{array}$ & 1 & 1 & 1 & - & LC - LC & $\begin{array}{l}\text { taperuçu-de-coleira- } \\
\text { branca }\end{array}$ \\
\hline $\begin{array}{c}\text { Chaetura cinereiventris (Sclater, } \\
\text { 1862) }\end{array}$ & 1 & 1 & 1 & - & LC - LC & $\begin{array}{l}\text { andorinhão-sobre- } \\
\text { cinzento }\end{array}$ \\
\hline $\begin{array}{c}\text { Chaetura meridionalis (Hellmayr, } \\
1907 \text { ) }\end{array}$ & 1 & 1 & 1 & - & LC - LC & andorinhão-do-temporal \\
\hline $\begin{array}{c}\text { Ramphodon naevius (Dumont, } \\
\text { 1818) }\end{array}$ & 0 & 0 & 1 & 1 & NT - LC & beija-flor-rajado \\
\hline $\begin{array}{c}\text { Phaethornis squalidus } \\
\text { (Temminck, 1822) }\end{array}$ & 1 & 1 & 0 & 1 & LC - LC & rabo-branco-pequeno \\
\hline
\end{tabular}




\begin{tabular}{|c|c|c|c|c|c|c|}
\hline Nome do Taxon & $\begin{array}{c}\text { Rebio } \\
\text { Sassafrás }\end{array}$ & $\begin{array}{l}\text { Gleba } \\
\text { Menor }\end{array}$ & $\begin{array}{c}\text { Rebio } \\
\text { Canela } \\
\text { Preta }\end{array}$ & END & IUCN -SC & Nome comum \\
\hline $\begin{array}{c}\text { Phaethornis eurynome (Lesson, } \\
\text { 1832) }\end{array}$ & 1 & 1 & 1 & 1 & LC - LC & garganta-rajada \\
\hline Florisuga fusca (Vieillot, 1817) & 0 & 0 & 1 & 1 & LC - LC & beija-flor-preto \\
\hline $\begin{array}{c}\text { Stephanoxis lalandi (Vieillot, } \\
\text { 1818) }\end{array}$ & 1 & 0 & 0 & 1 & LC - LC & beija-flor-de-topete \\
\hline $\begin{array}{c}\text { Chlorostilbon lucidus (Shaw, } \\
\text { 1812) }\end{array}$ & 1 & 1 & 0 & - & LC - LC & $\begin{array}{l}\text { besourinho-bico- } \\
\text { vermelho }\end{array}$ \\
\hline $\begin{array}{l}\text { Lophornis chalybeus (Vieillot, } \\
\text { 1822) }\end{array}$ & 0 & 1 & 0 & - & LC - LC & topetinho-verde \\
\hline $\begin{array}{l}\text { Thalurania glaucopis (Gmelin, } \\
\text { 1788) }\end{array}$ & 1 & 1 & 1 & 1 & LC - LC & beija-flor-de-fronte-violeta \\
\hline $\begin{array}{l}\text { Leucochloris albicollis (Vieillot, } \\
1818 \text { ) }\end{array}$ & 1 & 1 & 0 & 1 & LC - LC & beija-flor-de-papo-branco \\
\hline $\begin{array}{c}\text { Amazilia versicolor (Vieillot, } \\
\text { 1818) }\end{array}$ & 0 & 1 & 1 & - & LC - LC & $\begin{array}{l}\text { beija-flor-de-banda- } \\
\text { branca }\end{array}$ \\
\hline $\begin{array}{l}\text { Heliodoxa rubricauda (Boddaert, } \\
\text { 1783) }\end{array}$ & 1 & 1 & 1 & 1 & LC - LC & beija-flor-rubi \\
\hline $\begin{array}{c}\text { Calliphlox amethystina } \\
\text { (Boddaert, 1783) }\end{array}$ & 1 & 0 & 0 & - & LC - LC & estrelinha-ametista \\
\hline Trogon surrucura (Vieillot, 1817) & 1 & 1 & 1 & 1 & LC - LC & surucuá-variado \\
\hline Trogon rufus (Gmelin, 1788) & 1 & 1 & 1 & - & LC - LC & $\begin{array}{l}\text { surucuá-de-barriga- } \\
\text { amarela }\end{array}$ \\
\hline $\begin{array}{l}\text { Megaceryle torquata (Linnaeus, } \\
\text { 1766) }\end{array}$ & 0 & 1 & 0 & - & LC - LC & martim-pescador-grande \\
\hline $\begin{array}{l}\text { Chloroceryle amazona (Latham, } \\
\text { 1790) }\end{array}$ & 0 & 1 & 0 & - & LC - LC & martim-pescador-verde \\
\hline $\begin{array}{c}\text { Chloroceryle americana (Gmelin, } \\
\text { 1788) }\end{array}$ & 0 & 1 & 0 & - & LC - LC & $\begin{array}{l}\text { martim-pescador- } \\
\text { pequeno }\end{array}$ \\
\hline Malacoptila striata (Spix, 1824) & 0 & 0 & 1 & 1 & LC - LC & barbudo-rajado \\
\hline $\begin{array}{l}\text { Ramphastos dicolorus (Linnaeus, } \\
\text { 1766) }\end{array}$ & 1 & 1 & 1 & 1 & LC - LC & tucano-de-bico-verde \\
\hline $\begin{array}{c}\text { Selenidera maculirostris } \\
\text { (Lichtenstein, 1823) }\end{array}$ & 1 & 0 & 1 & 1 & LC - LC & araçari-poca \\
\hline $\begin{array}{l}\text { Pteroglossus bailloni (Vieillot, } \\
\text { 1819) }\end{array}$ & 1 & 0 & 1 & 1 & NT - LC & araçari-banana \\
\hline $\begin{array}{c}\text { Picumnus cirratus (Temminck, } \\
\text { 1825) }\end{array}$ & 1 & 0 & 0 & 1 & LC - LC & pica-pau-anão-barrado \\
\hline $\begin{array}{l}\text { Picumnus temminckii } \\
\text { (Lafresnaye, 1845) }\end{array}$ & 1 & 1 & 1 & 1 & LC - LC & pica-pau-anão-de-coleira \\
\hline $\begin{array}{l}\text { Picumnus nebulosus (Sundevall, } \\
\text { 1866) }\end{array}$ & 1 & 0 & 0 & 1 & NT - LC & pica-pau-anão-carijó \\
\hline $\begin{array}{c}\text { Melanerpes candidus (Otto, } \\
1796)\end{array}$ & 0 & 1 & 1 & - & LC - LC & pica-pau-branco \\
\hline $\begin{array}{c}\text { Melanerpes flavifrons (Vieillot, } \\
1818 \text { ) }\end{array}$ & 1 & 1 & 1 & 1 & LC - LC & $\begin{array}{l}\text { benedito-de-testa- } \\
\text { amarela }\end{array}$ \\
\hline $\begin{array}{c}\text { Veniliornis spilogaster (Wagler, } \\
1827 \text { ) }\end{array}$ & 1 & 1 & 1 & 1 & LC - LC & picapauzinho-verde-carijó \\
\hline
\end{tabular}




\begin{tabular}{|c|c|c|c|c|c|c|}
\hline Nome do Taxon & $\begin{array}{c}\text { Rebio } \\
\text { Sassafrás }\end{array}$ & $\begin{array}{l}\text { Gleba } \\
\text { Menor }\end{array}$ & $\begin{array}{c}\text { Rebio } \\
\text { Canela } \\
\text { Preta }\end{array}$ & END & IUCN -SC & Nome comum \\
\hline $\begin{array}{c}\text { Piculus aurulentus (Temminck, } \\
\text { 1821) }\end{array}$ & 1 & 1 & 1 & 1 & NT - LC & pica-pau-dourado \\
\hline $\begin{array}{c}\text { Colaptes melanochloros (Gmelin, } \\
1788)\end{array}$ & 1 & 0 & 0 & - & LC - LC & pica-pau-verde-barrado \\
\hline $\begin{array}{c}\text { Colaptes campestris (Vieillot, } \\
\text { 1818) }\end{array}$ & 1 & 1 & 1 & - & LC - LC & pica-pau-do-campo \\
\hline $\begin{array}{c}\text { Celeus flavescens (Gmelin, } \\
1788)\end{array}$ & 0 & 0 & 1 & - & LC - LC & pica-pau-cabeça-amarela \\
\hline $\begin{array}{c}\text { Celeus galeatus (Temminck, } \\
1822)\end{array}$ & 1 & 0 & 0 & 1 & VU - VU & pica-pau-de-cara-canela \\
\hline $\begin{array}{c}\text { Dryocopus lineatus (Linnaeus, } \\
1766 \text { ) }\end{array}$ & 1 & 1 & 1 & - & LC - LC & $\begin{array}{l}\text { pica-pau-de-banda- } \\
\text { branca }\end{array}$ \\
\hline $\begin{array}{c}\text { Campephilus robustus } \\
\text { (Lichtenstein, 1818) }\end{array}$ & 1 & 0 & 0 & - & LC - LC & pica-pau-rei \\
\hline $\begin{array}{c}\text { Myrmoderus squamosus Pelzeln, } \\
1868\end{array}$ & 1 & 1 & 1 & 1 & LC - LC & papa-formiga-de-grota \\
\hline Rhopias gularis (Spix, 1825) & 1 & 1 & 1 & 1 & LC - LC & $\begin{array}{l}\text { choquinha-garganta- } \\
\text { pintada }\end{array}$ \\
\hline $\begin{array}{l}\text { Myrmotherula unicolor } \\
\text { (Ménétriès, 1835) }\end{array}$ & 0 & 0 & 1 & 1 & NT - LC & choquinha-cinzenta \\
\hline $\begin{array}{l}\text { Dysithamnus stictothorax } \\
\text { (Temminck, 1823) }\end{array}$ & 0 & 0 & 1 & 1 & NT - LC & $\begin{array}{l}\text { choquinha-de-peito- } \\
\text { pintado }\end{array}$ \\
\hline $\begin{array}{l}\text { Dysithamnus mentalis } \\
\text { (Temminck, 1823) }\end{array}$ & 1 & 1 & 1 & - & LC - LC & choquinha-lisa \\
\hline $\begin{array}{l}\text { Herpsilochmus rufimarginatus } \\
\text { (Temminck, 1822) }\end{array}$ & 0 & 0 & 1 & - & LC - LC & $\begin{array}{l}\text { chorozinho-de-asa- } \\
\text { vermelha }\end{array}$ \\
\hline $\begin{array}{c}\text { Thamnophilus caerulescens } \\
\text { Vieillot, } 1816\end{array}$ & 1 & 1 & 1 & - & LC - LC & choca-da-mata \\
\hline $\begin{array}{c}\text { Thamnophilus ruficapillus } \\
\text { (Vieillot, 1816) }\end{array}$ & 1 & 0 & 0 & - & LC - LC & $\begin{array}{l}\text { choca-de-chapéu- } \\
\text { vermelho }\end{array}$ \\
\hline $\begin{array}{l}\text { Hypoedaleus guttatus (Vieillot, } \\
\text { 1816) }\end{array}$ & 0 & 1 & 1 & 1 & LC - LC & chocão-carijó \\
\hline Batara cinerea (Vieillot, 1819) & 1 & 1 & 1 & - & LC - LC & matracão \\
\hline $\begin{array}{c}\text { Mackenziaena leachii (Such, } \\
1825)\end{array}$ & 1 & 1 & 0 & 1 & LC - LC & borralhara-assobiadora \\
\hline $\begin{array}{c}\text { Mackenziaena severa } \\
\text { (Lichtenstein, 1823) }\end{array}$ & 1 & 1 & 1 & 1 & LC - LC & borralhara \\
\hline $\begin{array}{c}\text { Biatas nigropectus (Lafresnaye, } \\
1850 \text { ) }\end{array}$ & 1 & 0 & 0 & 1 & VU - VU & papo-branco \\
\hline Terenura maculata (Wied, 1831) & 0 & 0 & 1 & 1 & LC - LC & zidedê \\
\hline $\begin{array}{c}\text { Pyriglena leucoptera (Vieillot, } \\
\text { 1818) }\end{array}$ & 1 & 1 & 1 & 1 & LC - LC & papa-taoca-do-sul \\
\hline $\begin{array}{c}\text { Drymophila ferruginea } \\
\text { (Temminck, 1822) }\end{array}$ & 1 & 0 & 1 & 1 & LC - LC & trovoada \\
\hline $\begin{array}{l}\text { Drymophila rubricollis (Bertoni, } \\
\text { 1901) }\end{array}$ & 1 & 1 & 1 & - & LC - LC & trovoada-de-bertoni \\
\hline
\end{tabular}




\begin{tabular}{|c|c|c|c|c|c|c|}
\hline Nome do Taxon & $\begin{array}{c}\text { Rebio } \\
\text { Sassafrás }\end{array}$ & $\begin{array}{l}\text { Gleba } \\
\text { Menor }\end{array}$ & $\begin{array}{c}\text { Rebio } \\
\text { Canela } \\
\text { Preta }\end{array}$ & END & IUCN -SC & Nome comum \\
\hline $\begin{array}{c}\text { Drymophila ochropyga (Hellmayr, } \\
\text { 1906) }\end{array}$ & 0 & 0 & 1 & 1 & NT - LC & $\begin{array}{l}\text { choquinha-dorso- } \\
\text { vermelho }\end{array}$ \\
\hline $\begin{array}{c}\text { Drymophila malura (Temminck, } \\
\text { 1825) }\end{array}$ & 1 & 1 & 1 & 1 & LC - LC & choquinha-carijó \\
\hline $\begin{array}{c}\text { Conopophaga lineata (Wied, } \\
\text { 1831) }\end{array}$ & 1 & 1 & 1 & 1 & LC - LC & chupa-dente \\
\hline $\begin{array}{c}\text { Conopophaga melanops (Vieillot, } \\
\text { 1818) }\end{array}$ & 1 & 0 & 1 & 1 & LC - LC & $\begin{array}{l}\text { cuspidor-de-máscara- } \\
\text { preta }\end{array}$ \\
\hline Grallaria varia (Boddaert, 1783) & 1 & 1 & 0 & 1 & LC - LC & tovacuçu \\
\hline Hylopezus nattereri (Pinto, 1937) & 1 & 1 & 0 & 1 & LC - LC & pinto-do-mato \\
\hline Merulaxis ater (Lesson, 1830) & 1 & 0 & 0 & 1 & NT - LC & entufado \\
\hline $\begin{array}{l}\text { Eleoscytalopus indigoticus } \\
\text { (Wied, 1831) }\end{array}$ & 1 & 1 & 1 & 1 & NT - LC & macuquinho \\
\hline $\begin{array}{l}\text { Scytalopus speluncae } \\
\text { (Ménétriès, 1835) }\end{array}$ & 1 & 0 & 1 & 1 & LC - LC & tapaculo-preto \\
\hline $\begin{array}{l}\text { Psilorhamphus guttatus } \\
\text { (Ménétriès, 1835) }\end{array}$ & 1 & 1 & 1 & - & NT - LC & tapaculo-pintado \\
\hline $\begin{array}{l}\text { Chamaeza campanisona } \\
\text { (Lichtenstein, 1823) }\end{array}$ & 1 & 1 & 1 & - & LC - LC & tovaca-campainha \\
\hline $\begin{array}{c}\text { Chamaeza ruficauda (Cabanis \& } \\
\text { Heine, 1859) }\end{array}$ & 1 & 0 & 0 & 1 & LC - LC & tovaca-de-rabo-vermelho \\
\hline $\begin{array}{c}\text { Sclerurus scansor (Ménétriès, } \\
1835)\end{array}$ & 1 & 1 & 1 & 1 & LC - LC & vira-folha \\
\hline $\begin{array}{l}\text { Dendrocincla turdina } \\
\text { (Lichtenstein, 1820) }\end{array}$ & 1 & 1 & 1 & 1 & LC - LC & arapaçu-liso \\
\hline $\begin{array}{c}\text { Sittasomus griseicapillus (Vieillot, } \\
\text { 1818) }\end{array}$ & 1 & 1 & 1 & - & LC - LC & arapaçu-verde \\
\hline $\begin{array}{c}\text { Xiphorhynchus fuscus (Vieillot, } \\
\text { 1818) }\end{array}$ & 1 & 1 & 1 & 1 & LC - LC & arapaçu-rajado \\
\hline $\begin{array}{l}\text { Lepidocolaptes falcinellus } \\
\text { (Cabanis \& Heine, 1859) }\end{array}$ & 1 & 0 & 0 & 1 & LC - LC & arapaçu-escamado-do-sul \\
\hline $\begin{array}{c}\text { Campylorhamphus falcularius } \\
\text { (Vieillot, 1822) }\end{array}$ & 1 & 0 & 0 & - & LC - LC & arapaçu-de-bico-torto \\
\hline $\begin{array}{l}\text { Dendrocolaptes platyrostris } \\
\text { (Spix, 1825) }\end{array}$ & 1 & 1 & 1 & - & LC - LC & arapaçu-grande \\
\hline $\begin{array}{c}\text { Xiphocolaptes albicollis (Vieillot, } \\
\text { 1818) }\end{array}$ & 1 & 1 & 1 & - & LC - LC & $\begin{array}{l}\text { arapaçu-de-garganta- } \\
\text { branca }\end{array}$ \\
\hline $\begin{array}{c}\text { Xenops minutus (Sparrman, } \\
\text { 1788) }\end{array}$ & 0 & 0 & 0 & - & LC - LC & bico-virado-miúdo \\
\hline Xenops rutilans Temminck, 1821 & 1 & 1 & 1 & - & LC - LC & bico-virado-carijó \\
\hline $\begin{array}{l}\text { Clibanornis dendrocolaptoides } \\
\text { (Pelzeln, 1859) }\end{array}$ & 1 & 0 & 0 & 1 & LC - LC & cisqueiro \\
\hline Furnarius rufus (Gmelin, 1788) & 1 & 1 & 1 & - & LC - LC & joão-de-barro \\
\hline $\begin{array}{l}\text { Lochmias nematura } \\
\text { (Lichtenstein, 1823) }\end{array}$ & 1 & 1 & 1 & - & LC - LC & joão-porca \\
\hline $\begin{array}{c}\text { Anabazenops fuscus (Vieillot, } \\
1816 \text { ) }\end{array}$ & 1 & 0 & 1 & 1 & LC - LC & trepador-coleira \\
\hline
\end{tabular}




\begin{tabular}{|c|c|c|c|c|c|c|}
\hline Nome do Taxon & $\begin{array}{c}\text { Rebio } \\
\text { Sassafrás }\end{array}$ & $\begin{array}{l}\text { Gleba } \\
\text { Menor }\end{array}$ & $\begin{array}{c}\text { Rebio } \\
\text { Canela } \\
\text { Preta }\end{array}$ & END & IUCN -SC & Nome comum \\
\hline $\begin{array}{c}\text { Anabacerthia lichtensteini } \\
\text { (Cabanis \& Heine, 1859) }\end{array}$ & 0 & 0 & 1 & 1 & LC - LC & limpa-folha-ocráceo \\
\hline Philydor atricapillus (Wied, 1821) & 1 & 1 & 1 & 1 & LC - LC & limpa-folha-coroado \\
\hline Philydor rufum (Vieillot, 1818) & 1 & 1 & 1 & - & LC - LC & limpa-folha-de-testa-baia \\
\hline $\begin{array}{l}\text { Heliobletus contaminatus } \\
\text { (Berlepsch, } 1885\end{array}$ & 1 & 0 & 1 & 1 & LC - LC & trepadorzinho \\
\hline $\begin{array}{l}\text { Anabacerthia amaurotis } \\
\text { (Temminck, 1823) }\end{array}$ & 1 & 0 & 0 & 1 & NT - LC & limpa-folha-miúdo \\
\hline $\begin{array}{l}\text { Syndactyla rufosuperciliata } \\
\text { (Lafresnaye, 1832) }\end{array}$ & 1 & 1 & 1 & - & LC - LC & trepador-quiete \\
\hline $\begin{array}{l}\text { Cichlocolaptes leucophrus } \\
\text { (Jardine \& Selby, 1830) }\end{array}$ & 0 & 1 & 1 & 1 & LC - LC & trepador-sobrancelha \\
\hline $\begin{array}{l}\text { Leptasthenura setaria } \\
\text { (Temminck, 1824) }\end{array}$ & 1 & 0 & 0 & 1 & NT - LC & grimpeiro \\
\hline $\begin{array}{l}\text { Synallaxis ruficapilla Vieillot, } \\
1819\end{array}$ & 1 & 1 & 1 & 1 & LC - LC & Pichororé \\
\hline $\begin{array}{c}\text { Synallaxis cinerascens } \\
\text { Temminck, } 1823\end{array}$ & 1 & 0 & 0 & 1 & LC - LC & pi-puí \\
\hline Synallaxis spixi Sclater, 1856 & 1 & 1 & 1 & - & LC - LC & joão-teneném \\
\hline $\begin{array}{l}\text { Cranioleuca obsoleta } \\
\text { (Reichenbach, 1853) }\end{array}$ & 1 & 1 & 0 & 1 & LC - LC & arredio-oliváceo \\
\hline $\begin{array}{c}\text { Manacus manacus (Linnaeus, } \\
1766)\end{array}$ & 0 & 1 & 0 & - & LC - LC & rendeira \\
\hline $\begin{array}{l}\text { Ilicura militaris (Shaw \& Nodder, } \\
\text { 1809) }\end{array}$ & 1 & 1 & 0 & 1 & LC - LC & tangarazinho \\
\hline $\begin{array}{c}\text { Chiroxiphia caudata (Shaw \& } \\
\text { Nodder, 1793) }\end{array}$ & 1 & 1 & 1 & 1 & LC - LC & tangará \\
\hline $\begin{array}{c}\text { Oxyruncus cristatus Swainson, } \\
1821\end{array}$ & 1 & 0 & 0 & - & LC - LC & araponga-do-horto \\
\hline $\begin{array}{c}\text { Schiffornis virescens } \\
\text { (Lafresnaye, 1838) }\end{array}$ & 1 & 1 & 1 & 1 & LC - LC & flautim \\
\hline $\begin{array}{c}\text { Tityra inquisitor (Lichtenstein, } \\
1823 \text { ) }\end{array}$ & 1 & 0 & 1 & - & LC - LC & anambé-bochecha-parda \\
\hline Tityra cayana (Linnaeus, 1766) & 1 & 1 & 1 & - & LC - LC & anambé-de-rabo-preto \\
\hline $\begin{array}{l}\text { Pachyramphus viridis (Vieillot, } \\
\text { 1816) }\end{array}$ & 1 & 1 & 0 & - & LC - LC & caneleiro-verde \\
\hline $\begin{array}{l}\text { Pachyramphus castaneus } \\
\text { (Jardine \& Selby, 1827) }\end{array}$ & 1 & 1 & 1 & - & LC - LC & caneleiro \\
\hline $\begin{array}{c}\text { Pachyramphus polychopterus } \\
\text { (Vieillot, 1818) }\end{array}$ & 1 & 1 & 1 & - & LC - LC & caneleiro-preto \\
\hline $\begin{array}{c}\text { Pachyramphus validus } \\
\text { (Lichtenstein, 1823) }\end{array}$ & 1 & 1 & 0 & - & LC - LC & $\begin{array}{l}\text { caneleiro-de-chapéu- } \\
\text { preto }\end{array}$ \\
\hline $\begin{array}{c}\text { Procnias nudicollis (Vieillot, } \\
1817 \text { ) }\end{array}$ & 1 & 1 & 1 & 1 & VU - LC & araponga \\
\hline $\begin{array}{c}\text { Lipaugus lanioides (Lesson, } \\
1844 \text { ) }\end{array}$ & 0 & 0 & 1 & 1 & NT - EN & tropeiro-da-serra \\
\hline
\end{tabular}




\begin{tabular}{|c|c|c|c|c|c|c|}
\hline Nome do Taxon & $\begin{array}{c}\text { Rebio } \\
\text { Sassafrás }\end{array}$ & $\begin{array}{l}\text { Gleba } \\
\text { Menor }\end{array}$ & $\begin{array}{c}\text { Rebio } \\
\text { Canela } \\
\text { Preta }\end{array}$ & END & IUCN -SC & Nome comum \\
\hline $\begin{array}{c}\text { Carpornis cucullata (Swainson, } \\
\text { 1821) }\end{array}$ & 1 & 1 & 1 & 1 & NT - LC & corocochó \\
\hline $\begin{array}{c}\text { Phibalura flavirostris (Vieillot, } \\
\text { 1816) }\end{array}$ & 1 & 0 & 0 & - & NT - EN & tesourinha-da-mata \\
\hline $\begin{array}{l}\text { Platyrinchus mystaceus Vieillot, } \\
1818\end{array}$ & 1 & 1 & 1 & - & LC - LC & patinho \\
\hline $\begin{array}{l}\text { Platyrinchus leucoryphus Wied, } \\
1831\end{array}$ & 0 & 0 & 1 & 1 & VU - VU & patinho-gigante \\
\hline $\begin{array}{c}\text { Piprites pileata (Temminck, } \\
\text { 1822) }\end{array}$ & 1 & 0 & 0 & - & $\mathrm{VU}-\mathrm{EN}$ & caneleirinho-chapéu-preto \\
\hline $\begin{array}{l}\text { Mionectes rufiventris Cabanis, } \\
1846\end{array}$ & 1 & 1 & 1 & 1 & LC - LC & abre-asa-de-cabeça-cinza \\
\hline $\begin{array}{c}\text { Leptopogon amaurocephalus } \\
\text { Tschudi, } 1846\end{array}$ & 1 & 1 & 1 & - & LC - LC & cabeçudo \\
\hline $\begin{array}{c}\text { Phylloscartes ventralis } \\
\text { (Temminck, 1824) }\end{array}$ & 1 & 1 & 0 & - & LC - LC & borboletinha-do-mato \\
\hline $\begin{array}{c}\text { Tolmomyias sulphurescens } \\
\text { (Spix, 1825) }\end{array}$ & 1 & 1 & 1 & 1 & NT - LC & $\begin{array}{l}\text { bico-chato-de-orelha- } \\
\text { preta }\end{array}$ \\
\hline $\begin{array}{l}\text { Poecilotriccus plumbeiceps } \\
\text { (Lafresnaye, 1846) }\end{array}$ & 1 & 1 & 1 & - & LC - LC & tororó \\
\hline $\begin{array}{c}\text { Myiornis auricularis (Vieillot, } \\
\text { 1818) }\end{array}$ & 0 & 1 & 1 & 1 & LC - LC & miudinho \\
\hline $\begin{array}{l}\text { Hemitriccus obsoletus (Miranda- } \\
\text { Ribeiro, 1906) }\end{array}$ & 1 & 1 & 0 & 1 & LC - LC & Catraca \\
\hline $\begin{array}{c}\text { Hemitriccus orbitatus (Wied, } \\
\text { 1831) }\end{array}$ & 0 & 0 & 1 & 1 & NT - LC & tiririzinho-do-mato \\
\hline $\begin{array}{l}\text { Camptostoma obsoletum } \\
\text { (Temminck, 1824) }\end{array}$ & 1 & 1 & 1 & - & LC - LC & risadinha \\
\hline $\begin{array}{l}\text { Hirundinea ferruginea (Gmelin, } \\
\text { 1788) }\end{array}$ & 1 & 0 & 0 & - & LC - LC & gibão-de-couro \\
\hline Elaenia parvirostris Pelzeln, 1868 & 0 & 1 & 1 & - & LC - LC & guaracava-de-bico-curto \\
\hline $\begin{array}{c}\text { Elaenia mesoleuca (Deppe, } \\
\text { 1830) }\end{array}$ & 1 & 1 & 1 & - & LC - LC & tuque \\
\hline $\begin{array}{c}\text { Elaenia obscura (d'Orbigny \& } \\
\text { Lafresnaye, 1837) }\end{array}$ & 1 & 0 & 0 & - & LC - LC & tucão \\
\hline $\begin{array}{c}\text { Myiopagis caniceps (Swainson, } \\
\text { 1835) }\end{array}$ & 1 & 1 & 0 & - & LC - LC & guaracava-cinzenta \\
\hline $\begin{array}{l}\text { Tyranniscus burmeisteri } \\
\text { (Cabanis \& Heine, 1859) }\end{array}$ & 1 & 0 & 0 & - & LC - LC & piolhinho-chiador \\
\hline $\begin{array}{l}\text { Phyllomyias virescens } \\
\text { (Temminck, 1824) }\end{array}$ & 1 & 0 & 1 & - & LC - LC & piolhinho-verdoso \\
\hline $\begin{array}{l}\text { Phyllomyias fasciatus (Thunberg, } \\
\text { 1822) }\end{array}$ & 1 & 1 & 1 & - & LC - LC & piolhinho \\
\hline $\begin{array}{l}\text { Phyllomyias griseocapilla Sclater, } \\
1862\end{array}$ & 0 & 0 & 1 & 1 & NT - LC & piolhinho-serrano \\
\hline $\begin{array}{c}\text { Serpophaga subcristata (Vieillot, } \\
\text { 1817) }\end{array}$ & 1 & 0 & 0 & - & LC - LC & alegrinho \\
\hline
\end{tabular}




\begin{tabular}{|c|c|c|c|c|c|c|}
\hline Nome do Taxon & $\begin{array}{c}\text { Rebio } \\
\text { Sassafrás }\end{array}$ & $\begin{array}{l}\text { Gleba } \\
\text { Menor }\end{array}$ & $\begin{array}{c}\text { Rebio } \\
\text { Canela } \\
\text { Preta }\end{array}$ & END & IUCN -SC & Nome comum \\
\hline Attila phoenicurus Pelzeln, 1868 & 1 & 1 & 1 & - & LC - LC & capitão-castanho \\
\hline Attila rufus (Vieillot, 1819) & 0 & 1 & 1 & 1 & LC - LC & capitão-de-saíra \\
\hline $\begin{array}{l}\text { Legatus leucophaius (Vieillot, } \\
\text { 1818) }\end{array}$ & 1 & 0 & 1 & - & LC - LC & bem-te-vi-pirata \\
\hline $\begin{array}{l}\text { Ramphotrigon megacephalum } \\
\text { (Swainson, 1835) }\end{array}$ & 1 & 0 & 0 & - & LC - LC & maria-cabeçuda \\
\hline $\begin{array}{c}\text { Myiarchus swainsoni Cabanis \& } \\
\text { Heine, } 1859\end{array}$ & 1 & 1 & 1 & - & LC - LC & irré \\
\hline Sirystes sibilator (Vieillot, 1818) & 0 & 0 & 1 & - & LC - LC & gritador \\
\hline $\begin{array}{l}\text { Pitangus sulphuratus (Linnaeus, } \\
\text { 1766) }\end{array}$ & 1 & 1 & 1 & - & $\mathrm{LC}-\mathrm{LC}$ & bem-te-vi \\
\hline $\begin{array}{c}\text { Machetornis rixosa (Vieillot, } \\
\text { 1819) }\end{array}$ & 1 & 1 & 1 & - & LC - LC & suiriri-cavaleiro \\
\hline $\begin{array}{c}\text { Myiodynastes maculatus (Statius } \\
\text { Muller, 1776) }\end{array}$ & 1 & 1 & 1 & - & LC - LC & bem-te-vi-rajado \\
\hline $\begin{array}{c}\text { Megarynchus pitangua } \\
\text { (Linnaeus, 1766) }\end{array}$ & 1 & 1 & 0 & - & LC - LC & neinei \\
\hline Myiozetetes similis (Spix, 1825) & 1 & 0 & 0 & - & $\mathrm{LC}-\mathrm{LC}$ & bentevizinho \\
\hline $\begin{array}{l}\text { Tyrannus melancholicus Vieillot, } \\
1819\end{array}$ & 1 & 1 & 1 & - & LC - LC & suiriri \\
\hline Tyrannus savana Vieillot, 1808 & 1 & 1 & 0 & - & LC - LC & tesourinha \\
\hline $\begin{array}{l}\text { Empidonomus varius (Vieillot, } \\
\text { 1818) }\end{array}$ & 1 & 1 & 1 & - & LC - LC & peitica \\
\hline Colonia colonus (Vieillot, 1818) & 0 & 1 & 1 & - & LC - LC & viuvinha \\
\hline $\begin{array}{c}\text { Myiophobus fasciatus (Statius } \\
\text { Muller, 1776) }\end{array}$ & 1 & 1 & 1 & - & $\mathrm{LC}-\mathrm{LC}$ & filipe \\
\hline $\begin{array}{l}\text { Cnemotriccus fuscatus (Wied, } \\
\text { 1831) }\end{array}$ & 1 & 0 & 1 & - & LC - LC & guaracanuçu \\
\hline $\begin{array}{l}\text { Lathrotriccus euleri (Cabanis, } \\
\text { 1868) }\end{array}$ & 1 & 1 & 1 & - & LC - LC & enferrujado \\
\hline Contopus cinereus (Spix, 1825) & 1 & 1 & 0 & - & LC - LC & papa-moscas-cinzento \\
\hline $\begin{array}{c}\text { Satrapa icterophrys (Vieillot, } \\
\text { 1818) }\end{array}$ & 0 & 1 & 0 & - & LC - LC & suiriri-pequeno \\
\hline $\begin{array}{l}\text { Muscipipra vetula (Lichtenstein, } \\
1823 \text { ) }\end{array}$ & 1 & 1 & 0 & 1 & LC - LC & tesoura-cinzenta \\
\hline $\begin{array}{c}\text { Cyclarhis gujanensis (Gmelin, } \\
\text { 1789) }\end{array}$ & 1 & 1 & 1 & - & LC - LC & pitiguari \\
\hline Vireo chivi (Vieillot, 1817) & 1 & 1 & 1 & - & LC - LC & juruviara \\
\hline $\begin{array}{l}\text { Hylophilus poicilotis Temminck, } \\
1822\end{array}$ & 1 & 1 & 1 & - & LC - LC & verdinho-coroado \\
\hline $\begin{array}{c}\text { Cyanocorax chrysops (Vieillot, } \\
\text { 1818) }\end{array}$ & 1 & 1 & 0 & - & LC - LC & gralha-picaça \\
\hline $\begin{array}{c}\text { Cyanocorax caeruleus (Vieillot, } \\
\text { 1818) }\end{array}$ & 1 & 0 & 0 & - & NT - LC & gralha-azul \\
\hline $\begin{array}{c}\text { Pygochelidon cyanoleuca } \\
\text { (Vieillot, 1817) }\end{array}$ & 1 & 1 & 1 & - & LC - LC & $\begin{array}{c}\text { andorinha-pequena-de- } \\
\text { casa }\end{array}$ \\
\hline
\end{tabular}




\begin{tabular}{|c|c|c|c|c|c|c|}
\hline Nome do Taxon & $\begin{array}{c}\text { Rebio } \\
\text { Sassafrás }\end{array}$ & $\begin{array}{l}\text { Gleba } \\
\text { Menor }\end{array}$ & $\begin{array}{c}\text { Rebio } \\
\text { Canela } \\
\text { Preta }\end{array}$ & END & IUCN -SC & Nome comum \\
\hline $\begin{array}{c}\text { Stelgidopteryx ruficollis (Vieillot, } \\
1817 \text { ) }\end{array}$ & 1 & 1 & 1 & - & LC - LC & andorinha-serradora \\
\hline Progne tapera (Vieillot, 1817) & 1 & 1 & 0 & - & LC - LC & andorinha-do-campo \\
\hline Progne chalybea (Gmelin, 1789) & 1 & 1 & 1 & - & LC - LC & andorinha-doméstica \\
\hline $\begin{array}{c}\text { Tachycineta leucorrhoa (Vieillot, } \\
\text { 1817) }\end{array}$ & 1 & 1 & 1 & - & LC - LC & $\begin{array}{l}\text { andorinha-de-sobre- } \\
\text { branco }\end{array}$ \\
\hline $\begin{array}{l}\text { Troglodytes musculus } \\
\text { (Naumann, 1823) }\end{array}$ & 1 & 1 & 1 & - & LC - LC & corruíra \\
\hline Turdus flavipes (Vieillot, 1818) & 1 & 1 & 1 & - & $L C-L C$ & sabiá-una \\
\hline Turdus rufiventris (Vieillot, 1818) & 1 & 1 & 1 & - & LC - LC & sabiá-laranjeira \\
\hline $\begin{array}{c}\text { Turdus leucomelas (Vieillot, } \\
\text { 1818) }\end{array}$ & 0 & 1 & 1 & - & LC - LC & sabiá-barranco \\
\hline $\begin{array}{l}\text { Turdus amaurochalinus } \\
\text { (Cabanis, 1850) }\end{array}$ & 1 & 1 & 1 & - & LC - LC & sabiá-poca \\
\hline $\begin{array}{c}\text { Turdus subalaris (Seebohm, } \\
\text { 1887) }\end{array}$ & 1 & 0 & 0 & 1 & LC - LC & sabiá-ferreiro \\
\hline Turdus albicollis (Vieillot, 1818) & 1 & 1 & 1 & - & LC - LC & sabiá-coleira \\
\hline $\begin{array}{l}\text { Mimus saturninus (Lichtenstein, } \\
1823 \text { ) }\end{array}$ & 0 & 1 & 0 & - & LC - LC & sabiá-do-campo \\
\hline $\begin{array}{c}\text { Coereba flaveola (Linnaeus, } \\
\text { 1758) }\end{array}$ & 1 & 1 & 1 & - & LC - LC & cambacica \\
\hline $\begin{array}{l}\text { Saltator maxillosus (Cabanis, } \\
1851 \text { ) }\end{array}$ & 1 & 1 & 0 & 1 & $L C-L C$ & bico-grosso \\
\hline $\begin{array}{l}\text { Saltator fuliginosus (Daudin, } \\
1800 \text { ) }\end{array}$ & 1 & 0 & 1 & 1 & LC - VU & pimentão \\
\hline $\begin{array}{c}\text { Saltator similis (d'Orbigny \& } \\
\text { Lafresnaye, 1837) }\end{array}$ & 1 & 1 & 1 & - & LC - LC & trinca-ferro-verdadeiro \\
\hline $\begin{array}{c}\text { Poospiza thoracica (Nordmann, } \\
\text { 1835) }\end{array}$ & 1 & 0 & 0 & 1 & LC - LC & peito-pinhão \\
\hline $\begin{array}{c}\text { Orchesticus abeillei (Lesson, } \\
\text { 1839) }\end{array}$ & 1 & 1 & 0 & 1 & NT - LC & sanhaçu-pardo \\
\hline $\begin{array}{l}\text { Orthogonys chloricterus (Vieillot, } \\
\text { 1819) }\end{array}$ & 0 & 0 & 1 & 1 & LC - LC & catirumbava \\
\hline $\begin{array}{l}\text { Pyrrhocoma ruficeps (Strickland, } \\
\text { 1844) }\end{array}$ & 1 & 1 & 0 & 1 & LC - LC & cabecinha-castanha \\
\hline $\begin{array}{c}\text { Tachyphonus coronatus (Vieillot, } \\
\text { 1822) }\end{array}$ & 1 & 1 & 1 & 1 & LC - LC & tiê-preto \\
\hline $\begin{array}{c}\text { Trichothraupis melanops (Vieillot, } \\
\text { 1818) }\end{array}$ & 1 & 1 & 1 & - & LC - LC & tiê-de-topete \\
\hline $\begin{array}{c}\text { Tangara seledon (Statius Muller, } \\
1776)\end{array}$ & 1 & 1 & 1 & 1 & LC - LC & saíra-sete-cores \\
\hline $\begin{array}{c}\text { Tangara cyanocephala (Statius } \\
\text { Muller, 1776) }\end{array}$ & 1 & 1 & 1 & 1 & LC - LC & saíra-militar \\
\hline $\begin{array}{c}\text { Tangara desmaresti (Vieillot, } \\
\text { 1819) }\end{array}$ & 1 & 1 & 0 & 1 & $L C-L C$ & saíra-lagarta \\
\hline $\begin{array}{c}\text { Tangara sayaca (Linnaeus, } \\
\text { 1766) }\end{array}$ & 1 & 1 & 1 & - & LC - LC & sanhaçu-cinzento \\
\hline
\end{tabular}




\begin{tabular}{|c|c|c|c|c|c|c|}
\hline Nome do Taxon & $\begin{array}{c}\text { Rebio } \\
\text { Sassafrás }\end{array}$ & $\begin{array}{l}\text { Gleba } \\
\text { Menor }\end{array}$ & $\begin{array}{c}\text { Rebio } \\
\text { Canela } \\
\text { Preta } \\
\end{array}$ & END & IUCN -SC & Nome comum \\
\hline $\begin{array}{c}\text { Tangara cyanoptera (Vieillot, } \\
\text { 1817) }\end{array}$ & 1 & 1 & 1 & 1 & $\mathrm{NT}-\mathrm{LC}$ & sanhaçu-de-encontro-azul \\
\hline Tangara palmarum (Wied, 1823) & 0 & 1 & 0 & - & LC - LC & sanhaçu-do-coqueiro \\
\hline $\begin{array}{c}\text { Tangara ornata (Sparrman, } \\
\text { 1789) }\end{array}$ & 0 & 1 & 1 & 1 & LC - LC & $\begin{array}{l}\text { sanhaçu-encontro- } \\
\text { amarelo }\end{array}$ \\
\hline $\begin{array}{c}\text { Tangara preciosa (Cabanis, } \\
\text { 1850) }\end{array}$ & 1 & 1 & 0 & - & LC - LC & saíra-preciosa \\
\hline $\begin{array}{c}\text { Conirostrum speciosum } \\
\text { (Temminck, 1824) }\end{array}$ & 0 & 1 & 0 & - & LC - LC & $\begin{array}{l}\text { figuinha-de-rabo- } \\
\text { castanho }\end{array}$ \\
\hline $\begin{array}{c}\text { Stephanophorus diadematus } \\
\text { (Temminck, 1823) }\end{array}$ & 1 & 1 & 0 & - & LC - LC & sanhaçu-frade \\
\hline $\begin{array}{c}\text { Pipraeidea melanonota (Vieillot, } \\
1819)\end{array}$ & 1 & 1 & 1 & - & LC - LC & saíra-viúva \\
\hline Tersina viridis (Illiger, 1811) & 1 & 1 & 1 & - & LC - LC & saí-andorinha \\
\hline Dacnis cayana (Linnaeus, 1766) & 1 & 1 & 1 & - & LC - LC & saí-azul \\
\hline $\begin{array}{c}\text { Chlorophanes spiza (Linnaeus, } \\
1758)\end{array}$ & 0 & 0 & 1 & - & $L C-L C$ & saí-verde \\
\hline $\begin{array}{l}\text { Hemithraupis ruficapilla (Vieillot, } \\
\text { 1818) }\end{array}$ & 1 & 0 & 1 & - & LC - LC & saíra-ferrugem \\
\hline $\begin{array}{c}\text { Zonotrichia capensis (Statius } \\
\text { Muller, 1776) }\end{array}$ & 1 & 1 & 1 & - & LC - LC & tico-tico \\
\hline $\begin{array}{l}\text { Haplospiza unicolor (Cabanis, } \\
\text { 1851) }\end{array}$ & 1 & 1 & 0 & 1 & LC - LC & cigarra-bambu \\
\hline $\begin{array}{c}\text { Microspingus cabanisi } \\
\text { (Bonaparte, 1850) }\end{array}$ & 1 & 1 & 0 & - & LC - LC & tico-tico-da-taquara \\
\hline Sicalis flaveola (Linnaeus, 1766) & 1 & 1 & 1 & - & $L C-L C$ & $\begin{array}{l}\text { canário-da-terra- } \\
\text { verdadeiro }\end{array}$ \\
\hline $\begin{array}{c}\text { Volatinia jacarina (Linnaeus, } \\
\text { 1766) }\end{array}$ & 1 & 0 & 0 & - & LC - LC & Tiziu \\
\hline $\begin{array}{l}\text { Sporophila frontalis (Verreaux, } \\
\text { 1869) }\end{array}$ & 1 & 0 & 0 & 1 & $\mathrm{VU}-\mathrm{VU}$ & Pixoxó \\
\hline $\begin{array}{c}\text { Sporophila caerulescens (Vieillot, } \\
\text { 1823) }\end{array}$ & 1 & 1 & 1 & - & LC - LC & coleirinho \\
\hline $\begin{array}{c}\text { Amaurospiza moesta (Hartlaub, } \\
1853)\end{array}$ & 1 & 0 & 0 & 1 & NT - LC & negrinho-do-mato \\
\hline Tiaris fuliginosus (Wied, 1830) & 0 & 1 & 1 & - & LC - LC & cigarra-do-coqueiro \\
\hline Habia rubica (Vieillot, 1817) & 1 & 1 & 1 & - & LC - LC & tiê-do-mato-grosso \\
\hline $\begin{array}{c}\text { Cyanoloxia glaucocaerulea } \\
\text { (d'Orbigny \& Lafresnaye, 1837) }\end{array}$ & 1 & 0 & 0 & - & $L C-L C$ & Azulino \\
\hline $\begin{array}{l}\text { Setophaga pitiayumi (Vieillot, } \\
\text { 1817) }\end{array}$ & 1 & 1 & 1 & - & LC - LC & mariquita \\
\hline $\begin{array}{c}\text { Geothlypis aequinoctialis } \\
\text { (Gmelin, 1789) }\end{array}$ & 1 & 1 & 1 & - & LC - LC & pia-cobra \\
\hline $\begin{array}{l}\text { Basileuterus culicivorus (Deppe, } \\
\text { 1830) }\end{array}$ & 1 & 1 & 1 & 1 & LC - LC & pula-pula \\
\hline $\begin{array}{c}\text { Myiothlypis leucoblephara } \\
\text { (Vieillot, 1817) }\end{array}$ & 1 & 1 & 0 & - & LC - LC & pula-pula-assobiador \\
\hline Myiothlypis rivularis (Wied, 1821) & 0 & 0 & 1 & - & LC - LC & pula-pula-ribeirinho \\
\hline
\end{tabular}




\begin{tabular}{lcccccc}
\hline Nome do Taxon & $\begin{array}{c}\text { Rebio } \\
\text { Sassafrás }\end{array}$ & $\begin{array}{c}\text { Gleba } \\
\text { Menor }\end{array}$ & $\begin{array}{c}\text { Rebio } \\
\text { Canela } \\
\text { Preta }\end{array}$ & END & IUCN -SC & Nome comum \\
\hline $\begin{array}{c}\text { Cacicus chrysopterus (Vigors, } \\
\text { 1825) }\end{array}$ & 1 & 1 & 0 & - & LC - LC & Tecelão \\
$\begin{array}{c}\text { Molothrus rufoaxillaris Cassin, } \\
\text { 1866 }\end{array}$ & 0 & 1 & 0 & - & LC - LC & vira-bosta-picumã \\
$\begin{array}{c}\text { Molothrus bonariensis (Gmelin, } \\
\text { 1789) }\end{array}$ & 1 & 0 & 1 & - & LC - LC & vira-bosta \\
$\begin{array}{c}\text { Gnorimopsar chopi (Vieillot, } \\
\text { 1819) }\end{array}$ & 0 & 1 & 0 & - & LC - LC & graúna \\
$\begin{array}{c}\text { Pseudoleistes guirahuro (Vieillot, } \\
\text { 1819) }\end{array}$ & 0 & 1 & 0 & - & LC - LC & chopim-do-brejo \\
$\begin{array}{c}\text { Sporagra magellanica (Vieillot, } \\
\text { 1805) }\end{array}$ & 1 & 1 & 1 & - & LC - LC & pintassilgo \\
$\begin{array}{c}\text { Euphonia violacea (Linnaeus, } \\
\text { 1758) }\end{array}$ & 1 & 0 & 1 & - & LC - LC & gaturamo-verdadeiro \\
$\begin{array}{c}\text { Euphonia chalybea (Mikan, 1825) } \\
\text { Euphonia cyanocephala (Vieillot, } \\
\text { 1818) }\end{array}$ & 0 & 0 & 1 & - & LC - LC & cais-cais \\
$\begin{array}{c}\text { Euphonia pectoralis (Latham, } \\
\text { 1801) }\end{array}$ & 1 & 1 & 1 & - & LC - LC & gaturamo-rei \\
$\begin{array}{c}\text { 1822) } \\
\text { Chlorophonia (Thunberg, }\end{array}$ & 1 & 0 & 0 & - & LC - LC & gaturamo-bandeira \\
\hline & 237 & 190 & 182 & 102 & - & ferro-velho \\
\hline
\end{tabular}

\section{CONCLUSÃO}

Considerando a riqueza das áreas estudadas, o número de espécies endêmicas e com algum grau de ameaça, fica evidente a importância das Reservas Biológicas Sassafrás e Canela Preta para a conservação das espécies de aves florestais, especialmente no caso da gleba menor da Reserva Biológica do Sassafrás, que permanecia com sua avifauna pouco conhecida.

Estratégias integradas de conservação, como o manejo e o planejamento da paisagem em torno destas unidades, criando e mantendo corredores ecológicos, a ampliação das áreas ou a estruturação de mosaicos de unidades de conservação, podem ser importantes estratégias de proteção, não apenas para espécies de aves do domínio Floresta Atlântica.

Ainda, importante considerar que os resultados desse trabalho, especialmente considerando as estratégias sugeridas, poderão contribuir para os Objetivos do Desenvolvimento Sustentável - ODS 15 e, mais especificamente a meta 5 referente a "Tomar medidas urgentes e significativas para reduzir a degradação de habitat naturais, estancar a perda de biodiversidade e, até 2020, proteger e evitar a extinção de espécies ameaçadas".

\section{REFERENCES}

Albuquerque, J. L. B.; Brüggemann, F. M. 1996. A avifauna do Parque Estadual da Serra do Tabuleiro, Santa Catarina, Brasil e as implicações para a sua conservação. Acta Biológica Leopoldensia, 18(1): 47-68.

Almeida, Maria Elisa De Castro, Jacques Vielliard \& Manoel Martins Dias. 1999. Composição da avifauna em duas matas ciliares na bacia do rio Jacaré-Pepira, São Paulo, Brasil. Revista Brasileira de Zoologia. 16(4): 1087-1098.

Bege, L. A. R. \& Marterer, B. T. P. 1991. Conservação da avifauna na região sul do Estado de Santa Catarina. Florianópolis, SC: Fundação de Amparo à Tecnologia e Meio Ambiente (FATMA) 54 pp.

Bege, L. A.R. \& Pauli, B. T. 1989. As aves nas Ilhas Moleques do Sul - Santa Catarina. Aspectos da ecologia e anilhamento de aves marinhas. Florianópolis, Fatma. 64p.

Bierregaard, R. O. Lovejoy, T.1989. Effects of forest fragmentation on Amazonian understory bird communities. Acta Amazonica. 19, p. 215-241.

Bierregaard, R. O.; Lovejoy, T. E.; Kapos, V.; Santos, A. A \& R. Hutchings. 1992. The biological dynamics of tropical rain forest fragments. Bio Science 42:859-866. 
Bornschein, M. R. \& Reinert, B. L. 2000. Aves de três remanescentes florestais do norte do Estado do Paraná, sul do Brasil, com sugestões para a conservação e manejo. Revista Brasileira de Zoologia, 17(3): 615-636.

Boscolo, D. \& J. P. Metzger. 2011. Isolation determines patterns of species presence in highly fragmented landscapes. Ecography 34:1018-1029.

Boscolo, D. \& Metzger, J. P. 2009. Is bird incidence in Atlantic forest fragments influenced by landscape patterns at multiple scales? Landscape Ecology. 24:907 - 918.

Branco, J. O. 2000. Avifauna associada ao estuário do Saco da Fazenda, Itajaí, Santa Catarina, Brasil. Rev. Bras. Zool. 17: 387-394.

Branco, J. O. 2001. Descartes da pesca do camarãosete-barbas como fonte de alimento para aves marinhas. Rev. Bras. Zool. 18: 293-300.

Branco, J. O. 2003. Reprodução de aves marinhas em ilhas costeiras de Santa Catarina, Brasil. Rev. Bras. Zool. 20 (4): 619-623.

Branco, J. O. 2004. Reprodução de Sterna hirundinacea Lesson e S. eurygnatha Saunders (Aves, Laridae) no litoral de Santa Catarina, Brasil. Rev. Bras. Zool. 20 (4): 655-659.

Branco, J. O. e H. A. A. Fracasso. 2005. Ocorrência e abundância de Rynchops niger Linnaeus, no litoral de Santa Catarina, Brasil. Rev. Bras. Zool 22(2):430-432.

Brummelhaus, Jaqueline; Weber, Jessica; Petry, Maria Virginia. 2012. A influência da fragmentação da mata ciliar sobre a avifauna na Bacia Hidrográfica do Rio Caí, Rio Grande do Sul. Neotropical Biology \& Conservation, 7 (1): 57-66.

Cavarzere V, Marcondes Rs, Moraes Gp, Donatelli RJ. 2012. Comparação quantitativa da comunidade de aves de um fragmento de floresta semidecidual do interior do Estado de São Paulo em intervalo de 30 anos. Iheringia 102: 384-393.

Consema. 2011. Conselho Estadual do Meio Ambiente. Resolução 002 de 06 de dezembro de 2011 Reconhece a Lista Oficial de Espécies da Fauna Ameaçadas de Extinção no Estado de Santa Catarina e dá outras providências. Publicada no Diário Oficial - SC - No 19.237, de 20.12.2011. Páginas 2-8.

Cruz, A. C. E C. E, Zimmermann. 2011. As APPs Urbanas dentro de uma Estratégia de Conservação de Aves Florestais. Dynamis 16(2): 46.

D'angelo-Neto, S.; Venturin, N.; Oliveira-Filho, A. T. O. \& Costa, F. A. F. 1998. Avifauna de quatro fisionomias florestais de pequeno tamanho (5-8 ha) no Campus da UFLA. Revista Brasileira de Biologia. 58 (3): 463-472.
Dario, F.R. 2012. Avifauna em fragmento florestal localizado na região metropolitana de São Paulo. Atualidades Ornitológicas, 168:33-41.

Debinski, D.; Holt, R. D. 2000. Survey and overview of habitat fragmentation experiments. Conservation Biology. 114 (2): 342 - 365.

Franchin A. G.; Oliveira, G. M.; Melo, C.; Tomé, C. E. R. \& Marçal Júnior, O. 2004. Avifauna do Campus Umuarama, Universidade Federal de Uberlândia (Uberlândia, MG). Revista Brasileira Zoociências, 6 (2): 219-230.

Fundação Do Meio Ambiente (Santa Catarina) 2010. Plano de Manejo da Reserva Biológica Estadual do Sassafrás: Plano Básico: Projeto de Proteção da Mata Atlântica em Santa Catarina-PPMA, SC. Florianópolis, Socioambiental Consultores Associados. 174p.

Goerck, J. M. 1997. Patterns of rarity in the birds of the Atlantic Forest of Brazil. Conservation Biology. 11: 112-118.

Guztzazky, A. C; Cruz, A. C.; Rupp, A. E. \& Zimmermann, C. E. 2014. Comunidade de aves em um fragmento de Floresta Atlântica no bairro Fidélis, Blumenau, Santa Catarina, Brasil. Revista de Estudos Ambientais. 1: 101-112.

Krieck, C. 2006. Comunidade de aves silvestres no Parque Nacional da Serra do Itajaí. Trabalho de Conclusão de Curso. Universidade Regional de Blumenau. 70p.

Laps, R.R.; Cordeiro, P.H.C.; Kajiwara, D.; Ribon, R.; Rodrigues, A.A.F. \& Uejima, A. Aves. 2003. In: D.N. Ramboldi; D.A.S. Oliveira (ed.), Fragmentação de ecossistemas: causas, efeitos sobre a biodiversidade e recomendações de políticas públicas. MMA/SBF.153-158 pp.

Macarthur, R. H. \& Wilson, E.O. 1963. An equilibrium theory of insular zoogeography. Evolution. 17:373-387.

Macarthur, R. H. \& Wilson, E.O. 1967. The theory of island biogeography. Princeton University Press, Princeton.

Marenzi, R. C. \& Roderjan, C. V. 2005. Estrutura espacial da paisagem da morraria da Praia Vermelha (SC): subsídio à ecologia de paisagem. Floresta. 35 (2): 259-269.

Marsden, S. J., Whiffin, M. \& Galetti, M. 2001. Bird diversity and abundance in forest fragments and Eucalyptus plantations around an Atlantic forest reserve, Brazil. Biodiversity and Conservation. 10: 737-751.

Marterer, B. T. P. 1996. Avifauna do Parque Botânico Morro do Baú. Florianópolis, Fatma. 74p.

Matarazzo-Neuberger, W. M. 1995.Comunidade de cinco parques e pra-ças da Grande São Paulo, estado de São Paulo. Ararajuba, 3:13-19. 
Myers, N.; Mittermeier, R.A.; Mittermeier, C.; Fonseca, G.A. \& Kent, J. 2000. Biodiversity hotspot for conservation priorities. Nature. 403: 853-858.

Pacheco, J.F. \& Laps, R. R.2001.Notas sobre os primeiros registros de seis espécies de Suboscines de SC .Tangara 1(4):169-171.

Pardini, R. 2004. Effects of forest fragmentation on small mammals in an Atlantic Forest landscape. Biodiversity and conservation. 13(13): 2567-2586.

Piacentini, V.Q.; A. Aleixo, C.E. Agne, G.N. Maurício, J.F. Pacheco, G.A. Bravo, G.R.R. Brito, L.N. Naka, F. Olmos, S. Posso, L.F. Silveira, G.S. Betini, E. Carrano, I. FRANZ, A.C. LEES, L.M. LIMA, D. PIOLI, F. SCHUNCK, F.R. AMARAL, G.A. BENCKE, M. COHN-HAFT, L.F.A. FIGUEIREDO, F.C. STRAUBE \& E. CESARI. 2005. Lista comentada das aves do Brasil pelo Comitê Brasileiro de Registros Ornitológicos. Revista Brasileira de Ornitologia, 23(2): 91-298.

Pizo, M. A. 1997. Seed dispersal and predation in two populations of Cabralea canjerana(Meliaceae) in the Atlantic forest of southeastern Brazil. Journal of Tropical Ecology. 3:559-578. 1997.

Ribeiro, M.C., Metzger, J.P., Martensen, A.C., Ponzoni, F.J. \& Hirota, M.M. 2009. The Brazilian Atlantic Forest: How much is left, and how is the remaining forest distributed? Implications for conservation. Biological Conservation. 142: 1141-1153.

Roos, A. L. 2002. Aves de sub-bosque da Mata Atlântica litorânea em Santa Catarina. Tese de Mestrado. Universidade Federal de Minas Gerais. 93p.

Rosário, L. A. 1996. As Aves em Santa Catarina: distribuição geográfica e meio ambiente. Florianópolis. FATMA. $326 p$.

Rupp, A. E.; Thom-E-Silva; G.; Laps, R. R. \& Zimmermann, C. E. 2008. Novas Espécies de Aves para o Estado de SC, Sul do Brasil. Biotemas. 21(3): 163-168.

Rupp, A. E.; Laps, R. R. \& Zimmermann, C. E. 2007. Registros de Caprimulgiformes e a primeira ocorrência de Caprimulgus sericocaudatus (bacurau-rabo-de-seda) no Estado de SC, Brasil. Revista Brasileira de Ornitologia. 15(4): $63-16$.

Sick, H. 1997. Ornitologia brasileira. Rio de Janeiro. Nova Fronteira. 912p.

Sick, H.; Bege, L. A. R. 1984. Novas informações sobre as aves do Estado de Santa Catarina. Anais da Sociedade Rio Grandense de Ornitologia. Porto Alegre. 5:3-6.

Sick, H., Azevedo, T. R.; Bege, L. A. R \& Voss, W. A. 1979b. Lista preliminar das aves existentes nos Parques e Reservas Biológicas de Santa Catarina. Projeto "Aves das ilhas do litoral catarinense" UFSC/FATMA, Florianópolis. pp. 31-40.
Sick, H., Azevedo T. R \& Bege, L. A. R. 1979a. Lista preliminar das aves do Estado de Santa Catarina. FATMA, Florianópolis. 6 pp.

Sick, H.; Bege, L. A. R.; Azevedo, T. R.1981. Aves do Estado de Santa Catarina. FATMA Sellowia, Série Zoologia. Florianópolis, (1):1-51.

Sick, H., Bege, L. A. R. \& Pauli, B. T. 1988. As aves do município de Antônio Carlos, pp. 426-431. In: Reitz, R. (ed.) Alto Biguaçu: narrativa cultural tetraracial. Florianópolis: Editora Lunardelli/Editora da UFSC.

Santos, M.P.D., Cerqueira, P.V.; Soares, L.M.S. 2010. Avifauna em seis localidades no centro-sul do Estado do Maranhão, Brasil. Ornithologia, 4(1): 49-65.

Soares, M. \& Schieffler, A. F. 1995a. Aves da ilhota da Galheta, Laguna, Sc, Brasil. Arq. Biol. Tecnologia. 38(4): $1101-1107$.

Soares, M. \& Schieffler, A. F. 1995b. Reprodução de Larus dominicanus (Aves, Laridae), na ilhota da Galheta, Laguna, Sc, Brasil. Arq. Biol. Tecnologia. 38(1): 313- 316.

Straube, F.C. \& Urben-Filho, A. 2004. Uma revisão crítica sobre o grau de conhecimento da avifauna do Parque Nacional do Iguaçu (Paraná, Brasil) e áreas adjacentes. Atualidades Ornitológicas 118:6. Publicação online disponível na íntegra em http:// www.ao.com.br (seção AO-Online).

Straube, F. C. \& Bianconi, G. V. 2002. Sobre a grandeza e a unidade utilizada para estimar esforço de captura com utilização de redes-deneblina. Chiroptera Neotropical. 8(1-2): 150-152.

Straube, F. C.; A. Urben-Filho \& D. Kajiwara. 2004. Aves. In: S. B. Mikish \& R. S. Bérnils (Eds). Livro vermelho da fauna Ameaçada de Extinção no Estado do Paraná. Curitiba: Instituto Ambiental do Paraná.143-496pp.

Tabanez, A.J.; Viana, M.V. \& Dias, A. S. 1997. Consequências da fragmentação e do efeito de borda sobre a estrutura, diversidade e sustentabilidade de um fragmento de floresta de planalto de Piracicaba. Revista Brasileira de Biologia. 57 (1): 47-60.

Tonetti, V.R \& Cavarzere, V. 2017. Betadiversity analysis of a bird assemblage of a biodiversity hotspot within the Atlantic Forest. Ornithologia Neotropical, 28: 281-290.

Valadão, R. M.; Franchin, A. G. \& Marçal Júnior, O. 2006. A avifauna no Parque Municipal Santa Luzia, zona urbana de Uberlândia, Minas Gerais. Revista Bioscience Journal, 22 (2): 97-102.

Zimmermann, C. E. 1989. Levantamento Preliminar da Ornitofauna da Mata de Galeria do Itajaí-Açú. Revista de Divulgação Cultural da FURB. 40 (12):51-62. 
Zimmermann, C. E. 1992. Uma contribuição à ornitologia catarinense - levantamento preliminar da ornitofauna do Parque Ecológico Artex. Dynamis. 1(1): 69-80.

Zimmermann, C. E. 1993. Nota sobre a avifauna do Parque Ecológico Spitzkopf. Dynamis.1(3): 7-13.

Zimmermann, C. E. 1995. Novas informações sobre a avifauna do Parque Ecológico Artex. Biotemas. $8(1): 7-20$.

Zimmermann, C. E. 1999.Avifauna de um fragmento de Floresta Atlântica em Blumenau, SC. Revista de Estudos Ambientais 1(3): 101-112.

Zimmermann, C. E. \& Branco, J. O. 2009. Avifauna associada aos fragmentos florestais do Saco da Fazenda. In: Branco J. O. (ed.). Estuário do Rio Itajaí-Açú, Santa Catarina: caracterização ambiental e alterações antrópicas. Itajaí: Editora UNIVALI. 263-272 pp.

Zimmermann, C. E., Piazera, M., Dambrowski, V. \& Silva Junior, L. A.S. 2017. Ecologia da Paisagem como Ferramenta de Análise para a Conservação da Biodiversidade nos Municípios De Itajaí e Navegantes, SC, Brasil. Braz. J. Aquat. Sci. Technol. 21 (2): 26-32.

Zimmermann, C. E., Muller, J. A. \& Cordeiro, R. O. 2018. Levantamento da Avifauna. In: Diniz, M. V., Filho, E. A. (eds.). Diagnóstico Prévio: subsídio preliminar para projetos de restauração ecossistêmica: Edifurb. 59-83.

Submetido: Setembro/19 Revisado: Setembro/19 Aceito: Outubro/19 Publicado: 17/04/2020 